\title{
Novel Polyphenols That Inhibit Colon Cancer Cell Growth Affecting Cancer Cell Metabolism $\$$
}

\author{
Marta Gómez de Cedrón, Teodoro Vargas, Andrés Madrona, ${ }^{1}$ Aranza Jiménez, ${ }^{2}$ \\ María-Jesús Pérez-Pérez, José-Carlos Quintela, Guillermo Reglero, Ana San-Félix, \\ and Ana Ramírez de Molina
}

Molecular Oncology and Nutritional Genomics of Cancer, IMDEA-Food Institute, CEI UAM+CSIC, Madrid, Spain (M.G.d.C., T.V., G.R., A.R.d.M.); Instituto de Química Médica (IQM, CSIC), Juan de la Cierva 3, Madrid, Spain (A.M., A.J., M.-J.P.-P., A.S.-F.); and Natac Biotech S.L., Parque Científico de Madrid, Campus de Cantoblanco, Madrid, Spain (J.-C.Q.)

Received April 19, 2018; accepted June 1, 2018

\begin{abstract}
New series of polyphenols with a hydrophilic galloyl-based head and a hydrophobic $N$-acyl tail, linked through a serinol moiety, have been synthesized and tested against colon cancer cell growth. Our structure activity relationship studies revealed that galloyl moieties are essential for growth inhibition. Moreover, the length of the $N$-acyl chain is crucial for the activity. Introduction of a $(Z)$ double bond in the acyl chain increased the anticancer properties. Our findings demonstrate that 16, the most potent compound within this series, has inhibitory effects on colon cancer cell growth and metabolism (glycolysis and mitochondrial
\end{abstract}

respiration) at the same time that it activates 5'AMP-activated kinase (AMPK) and induces apoptotic cell death. Based on these results, we propose that $\mathbf{1 6}$ might reprogram colon cancer cell metabolism through AMPK activation. This might lead to alterations on cancer cell bioenergy compromising cancer cell viability. Importantly, these antiproliferative and proapoptotic effects are selective for cancer cells. Accordingly, these results indicate that 16, with an unsaturated C18 chain, might be a useful prototype for the development of novel colon cancer cell growth inhibitors affecting cell metabolism.

\section{Introduction}

In recent years, the understanding of differences in cell metabolism between normal and cancer cells has been considered essential in the design and development of new anticancer drugs. In this respect, proliferating cancer cells exhibit a higher dependency on aerobic glycolysis than normal cells (Lopez-Lazaro, 2010; Marchetti et al., 2014), a phenomenon known as the Warburg effect (Warburg, 1956). This metabolic difference has led to the hypothesis that inhibition of glycolysis may preferentially kill cancer cells and may have

Authors declare no potential conflict of interest.

Copies of ${ }^{1} \mathrm{H}$ and ${ }^{13} \mathrm{C}$ NMR spectra are included in the supplemental material.

This work was supported by the Spanish Plan Nacional de Cooperación Público-Privada, Subprograma INNPACTO (IPT-2012-0213 060000, cofinanced by the FEDER program); the Spanish MINECO [Project SAF201564629-C2-1-R (MINECO/FEDER)]; AGL2016-76736-C3-3-R (MINECO/FEDER); and Regional government Comunidad de Madrid (P2013/ABI-2728, ALIBIRD-CM) and European Union Structural Funds. The European Social Fund (Youth Employment Operational Program 2014-2020) supports a contract in practices (to A.J.).

A.M. and T.V. contributed equally to this work.

${ }^{1}$ Current affiliation: Novartis Farmacéutica S.A., Barcelona, Spain.

${ }^{2}$ Current affiliation: Agencia Española de Medicamentos y Productos Sanitarios, Madrid, Spain.

https://doi.org/10.1124/jpet.118.248278.

S This article has supplemental material available at jpet.aspetjournals.org. significant therapeutic implications (Pelicano et al., 2006). Indeed, several glycolytic inhibitors (2-deoxy-D-glucose, lonidamine, 3-bromopyruvate, and dichloroacetate) have shown anticancer activities both in vitro and in vivo, and some of them have entered clinical trials. However, they show important drawbacks (low potency, instability...) that may limit their use as therapeutic drugs. Thus, development of new generations of glycolytic inhibitors with high potency, chemical stability, and good safety profiles represents an important task in the anticancer field.

In addition, cancer cells and normal cells metabolize oxygen $\left(\mathrm{O}_{2}\right)$ differently. Normal cells use oxygen $\left(\mathrm{O}_{2}\right)$ to generate energy in the form of ATP at mitochondria; meanwhile, cancer cells use $\mathrm{O}_{2}$ to generate high levels of reactive oxygen species, and aerobic glycolysis is used to provide energy and satisfy structural demands. This difference in the metabolism of $\mathrm{O}_{2}$ can also be exploited to kill cancer cells selectively (Marchetti et al., 2014).

Lately, natural and synthetic polyphenols have become a focus of interest on cancer chemoprevention and chemotherapy. In fact, epidemiologic studies suggest that the intake of polyphenols may protect against tumor growth (Neuhouser, 2004; Arts and Hollman, 2005), particularly gastrointestinal cancers (Pierini et al., 2008). The growth inhibition induced by polyphenols in tumor cells includes several inhibitory

ABBREVIATIONS: AMPK, 5'AMP-activated kinase; DMAP, dimethylaminopyridine; DMSO, dimethylsulfoxide; ECAR, extracellular acidification rate; FCCP, cyanide-4-(trifluoromethoxy)phenylhydrazone; HATU, (1-[bis(dimethylamino)methylene]-1H-1,2,3-triazolo[4,5-b]pyridinium 3-oxid hexafluorophosphate); MOM, methoxymethyl; MTT, 3-(4,5-dimethylthiazol-2-yl)-2,5-diphenyltetrazolium bromide; OCR, oxygen consumption rate; P-AMPK, phosphorylated form of AMPK; THF, tetrahydrofurane. 
mechanisms such as the activation of caspases, cell cycle arrest, and the inhibition of survival or proliferative signaling pathways (nuclear factor $\kappa \mathrm{B}$; Janus kinase/signal transducer and activator of transcription), among others (Hadi et al., 2007; Gonzalez-Vallinas et al., 2013; Zhang et al., 2015; Kubatka et al., 2016).

Moreover, there are many examples of dietary polyphenols affecting different metabolic pathways of cancer cells (Cerella et al., 2013). Flavonoids, quercetin, oleuropein, hydroxytyrosol, and epigallocatechin-3-gallate have been shown to affect glycolysis-related factors; meanwhile, others like curcumin or resveratrol target mitochondrial metabolism, inducing cell death and/or apoptosis in a variety of cancer models (Kim et al., 2009; Teiten et al., 2010; Boyer et al., 2012). In many cases, the underlying mechanism is associated with 5'AMPactivated kinase (AMPK) activation (Shin et al., 2009; Hung, et al., 2012).

AMPK is a central metabolic sensor that regulates anabolism or catabolism in response to energetic demands and/or oxidative stress. When activated, AMPK inhibits the main anabolic pathways that promote cell growth (Hardie, 2014; Li et al., 2015). In cancer, in which the energetic demands are elevated to support rapid cell growth and division, AMPK activators can be suitable candidates for therapeutic intervention. In fact, AMPK is emerging as a promising target for cancer prevention and therapy (Luo et al., 2010).

As a part of an ongoing work in our laboratories directed to the synthesis and biologic evaluation of polyphenols as antiviral and antibacterial agents (Flores et al., 2014; Rivero-Buceta et al., 2015a,b), we prepared molecules of general formula I, with a hydrophilic head, composed of two galloyl (3,4,5-trihydroxybenzoyl) units $\left(\mathrm{R}^{1}\right)$, and a hydrophobic tail (R), composed of an $N$-acyl-chain, linked through a serinol moiety (Fig. 1). Systematic modifications have been performed in these compounds. First, the effect of the $N$-acyl chain length of the hydrophobic tail was analyzed by introduction of different aliphatic acyl residues with variable number of carbons $(\mathrm{C} 2, \mathrm{C} 7, \mathrm{C} 11$, and $\mathrm{C} 17)$. The effect of unsaturation (one or two double bonds) on the hydrophobic tail was also analyzed. Modifications on the head were also performed by replacing the galloyl moieties, with three $\mathrm{OHs}$, by other phenolic units with two OHs. Finally, ethanolamine, instead of serinol, was used as a linker.

For all of these compounds, inhibition of colon cancer cell proliferation and colon cancer energetic metabolism (glycolysis and oxygen consumption), together with activation of AMPK and induction of apoptotic cell death, have been determined and are described in this work.

\section{Materials and Methods}

\section{Synthesis}

General Methods. Commercial reagents and solvents were used as received from the suppliers without further purification, unless otherwise stated. Dichloromethane was dried prior to use by distillation from $\mathrm{CaH}_{2}$ and stored over Linde type activated $4 \AA$ molecular sieves.

Analytical thin-layer chromatography was performed on aluminum plates precoated with silica gel $60\left(\mathrm{~F}_{254}, 0.25 \mathrm{~mm}\right)$. Products were visualized using a UV lamp (254 and $365 \mathrm{~nm}$ ) or by heating on a hot plate (approximately $200^{\circ} \mathrm{C}$ ), directly or after treatment with a $5 \%$ solution of phosphomolybdic acid or vanillin in ethanol.

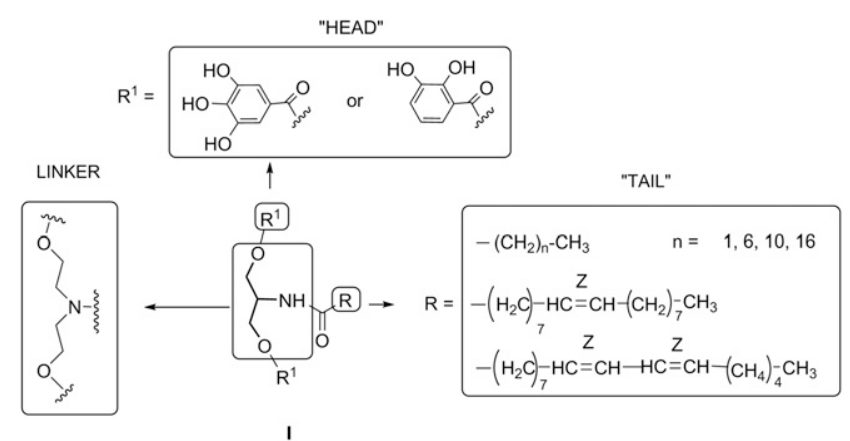

Fig. 1. Target polyphenols with two heads and one tail.

The compounds were purified by the following: 1) flash column chromatography on silica gel (60 Merck 230-400 mesh) and 2) trituration with cold diethyl ether and dichloromethane.

NMR spectra $\left({ }^{1} \mathrm{H},{ }^{13} \mathrm{C}\right.$ NMR) were recorded on a Varian UNIT INOVA-300 (300 MHz), Bruker AVANCE 300 (300 and $75 \mathrm{MHz}$ ), Varian INOVA-400 (400 and $100 \mathrm{MHz}$ ), Varian MERCURY-400 (400 and $100 \mathrm{MHz}$ ), and Varian-500 (500 and $125 \mathrm{MHz}$ ) spectrometers, using DMSO- $d_{6}$ and $\mathrm{CDCl}_{3}$ as solvents. Chemical shift $(\delta)$ values are reported in ppm relative to tetramethylsilane in ${ }^{1} \mathrm{H}$ and $\mathrm{CDCl}_{3}(\delta=77.0)$ in ${ }^{13} \mathrm{C}$ NMR. Coupling constants ( $J$ values) are reported in $\mathrm{Hz}$, and multiplicities of signals are indicated by the following symbols: $\mathrm{s}$ (singlet), $\mathrm{d}$ (doublet), $\mathrm{t}$ (triplet), $\mathrm{q}$ (quadruplet), m (multiplet), and bs (broad singlet). Some twodimensional spectra (COSY, HSQC, and HMBC) were performed to identify the structure.

Final compounds were lyophilized using a Telstar 6-80 system.

General Procedure for the Synthesis of Serinol Derivates 2-6. A stirred solution of 2-amino-1,3-propanediol (serinol) 1 (1 eq) and triethylamine in $\mathrm{MeOH}(10 \mathrm{ml})$ was cooled at $-20^{\circ} \mathrm{C}$ and treated dropwise with a solution of the corresponding acyl chloride (1.1 eq) in tetrahydrofurane (THF) $(5 \mathrm{ml})$. The reaction mixture was allowed to warm to room temperature and stirred overnight. Then it was poured into brine and extracted with dichloromethane $(3 \times 10 \mathrm{ml})$. The combined organic phases were washed with brine, dried over $\mathrm{MgSO}_{4}$, and concentrated under reduced pressure. The residue was purified by flash column chromatography on silica gel using as eluent EtOAc/ $\mathrm{MeOH}$ (10:1 to $7: 1$ ) to provide the corresponding $N$-acyl serinol derivatives.

N-Propanoylserinol (2). According to the general procedure, serinol (150 mg, $1.64 \mathrm{mmol}$ ) was treated with propanoyl chloride $(1.86 \mathrm{mmol}, 0.16 \mathrm{ml})$ and triethylamine $(0.4 \mathrm{ml})$ to give the title compound (202 mg, 81\%) as an amorphous white solid. ${ }^{1} \mathrm{H}$ NMR [400 $\mathrm{MHz}$, dimethylsulfoxide (DMSO)- $\left.d_{6}\right] \delta 7.40(\mathrm{~d}, J=8.1 \mathrm{~Hz}, 1 \mathrm{H}, \mathrm{NH})$, $4.55(\mathrm{t}, J=5.5 \mathrm{~Hz}, 2 \mathrm{H}, \mathrm{OH}), 3.74-3.64(\mathrm{~m}, 1 \mathrm{H}, \mathrm{CH}), 3.37(\mathrm{t}, J=5.6 \mathrm{~Hz}$, $\left.4 \mathrm{H}, \mathrm{CH}_{2} \mathrm{OH}\right), 2.06\left(\mathrm{t}, J=7.4 \mathrm{~Hz}, 2 \mathrm{H}, \mathrm{CH}_{2} \mathrm{C}=\mathrm{O}\right), 1.51-1.42(\mathrm{~m}, 2 \mathrm{H}$, $\left.\mathrm{CH}_{2}\right), 1.30-1.15\left(\mathrm{~m}, 8 \mathrm{H}, \mathrm{CH}_{2}\right), 0.86\left(\mathrm{t}, J=6.8 \mathrm{~Hz}, 3 \mathrm{H}, \mathrm{CH}_{3}\right)$.

N-Dodecanoyl Serinol (3). According to the general procedure, serinol (200 mg, $2.19 \mathrm{mmol}$ ) was treated with dodecanoyl chloride $(625 \mathrm{mg}, 2.85 \mathrm{mmol})$ and triethylamine $(0.6 \mathrm{ml})$ to give the title compound $(478 \mathrm{mg}, 80 \%)$ as an amorphous white solid. Characterization of this compound is consistent with those found in the literature (Kim et al., 2009; Boyer et al., 2012).

N-Octadecanoyl Serinol (4). According to the general procedure, serinol (150 mg, $1.64 \mathrm{mmol})$ was treated with octadecanoyl chloride $(545.24 \mathrm{mg}, 1.80 \mathrm{mmol})$ and triethylamine $(0.4 \mathrm{ml})$ to give the title compound ( $421 \mathrm{mg}, 75 \%)$ as an amorphous white solid. Characterization of this compound is consistent with those found in the literature (Bieberich et al., 2002a; Schulz and Robins, 2004; Merg et al., 2015).

N-Oleoyl Serinol (5). Serinol (55 mg, $0.60 \mathrm{mmol}$ ) was treated with 9-octadecenoyl chloride (oleoyl chloride) $(198.6 \mathrm{mg}, 0.66 \mathrm{mmol})$ and triethylamine $(0.1 \mathrm{ml})$ to give the title compound $(149 \mathrm{mg}, 70 \%)$ as an amorphous white solid. Characterization of this compound is 
consistent with those found in the literature (Bieberich et al., 2002a; Osornio et al., 2012; Landau and Siegel, 2014).

N-Linoleoyl Serinol (6). Serinol (55 mg, $1.64 \mathrm{mmol}$ ) was treated with linoleoyl chloride $(1.81 \mathrm{mmol}, 0.6 \mathrm{ml})$ and triethylamine $(0.1 \mathrm{ml})$ to give the title compound ( $169 \mathrm{mg}, 79 \%$ ) as an amorphous white solid. Characterization of this compound in the literature was incomplete (Oette and Tschung, 1980). Missing data (NMR) was now included. ${ }^{1} \mathrm{H}$ NMR (400 MHz, DMSO-d $\left.{ }_{6}\right) \delta 7.41(\mathrm{~d}, J=8.1 \mathrm{~Hz}, 1 \mathrm{H}, \mathrm{NH}), 5.35-5.27$ $(\mathrm{m}, 2 \mathrm{H}, \mathrm{CH}=\mathrm{CH}), 4.56(\mathrm{t}, J=5.5 \mathrm{~Hz}, 2 \mathrm{H}, \mathrm{OH}), 3.74-3.63(\mathrm{~m}, 1 \mathrm{H}, \mathrm{CH}$, $\mathrm{NH}), 3.38\left(\mathrm{t}, J=6.0 \mathrm{~Hz}, 4 \mathrm{H}, \mathrm{CH}_{2} \mathrm{OH}\right), 2.05\left(\mathrm{t}, J=7.5 \mathrm{~Hz}, 2 \mathrm{H}, \mathrm{CH}_{2} \mathrm{C}=\right.$ O), 2.01-1.92 (m, 4H, $\left.\mathrm{CH}_{2} \mathrm{HC}=\mathrm{CHCH}_{2}\right), 1.50-1.42\left(\mathrm{~m}, 2 \mathrm{H}, \mathrm{CH}_{2}\right)$, 1.34-1.14 (m, 22H, $\left.\mathrm{CH}_{2}\right), 0.85\left(\mathrm{t}, J=6.8 \mathrm{~Hz}, 3 \mathrm{H}, \mathrm{CH}_{3}\right)$.

3,4,5-Tris(Methoxymethoxy)Benzoic Acid (7). To a cooled $\left(0^{\circ} \mathrm{C}\right)$ mixture of methylgallate $(500 \mathrm{mg}, 2.71 \mathrm{mmol})$ and $N, N$ diisopropylethylamine $(1.6 \mathrm{ml}, 8.96 \mathrm{mmol})$ in dichloromethane $(20 \mathrm{ml})$ was added methoxymethyl $(\mathrm{MOM})$ chloride $(0.7 \mathrm{ml}, 8.97 \mathrm{mmol})$. The reaction was stirred at $0^{\circ} \mathrm{C}$ for 20 minutes and then at room temperature overnight. The solvent was evaporated to dryness, and the residue was dissolved in ethyl acetate $(20 \mathrm{ml})$ and washed successively with a saturated solution of ammonium chloride $(3 \times$ $20 \mathrm{ml})$ and water $(3 \times 20 \mathrm{ml})$. The organic layer was dried over anhydrous $\mathrm{Na}_{2} \mathrm{SO}_{4}$, filtered, and concentrated under vacuum. The residue was then purified by flash column chromatography using hexane/EtOAc (6:1 to 2:1) as the eluent to give $543 \mathrm{mg}$ (63\%) of methyl 3,4,5-tris(methoxymethoxy)benzoate as a white amorphous solid that was used for the next step. ${ }^{1} \mathrm{H}-\mathrm{NMR}\left(300 \mathrm{MHz}, \mathrm{CD}_{3} \mathrm{Cl}\right) \delta 7.51(\mathrm{~s}, 2 \mathrm{H}$, $\mathrm{H}-\mathrm{Ph}), 5.24$ (s, $\left.4 \mathrm{H}, 2 \times \mathrm{CH}_{2} \mathrm{MOM}\right), 5.13\left(\mathrm{~s}, 2 \mathrm{H}, 1 \times \mathrm{CH}_{2} \mathrm{MOM}\right), 3.83$ (s, $3 \mathrm{H}, \mathrm{OCH}_{3}$ ), 3.51 (s, 3H, $\left.1 \times \mathrm{CH}_{3} \mathrm{MOM}\right), 3.41\left(\mathrm{~s}, 6 \mathrm{H}, 2 \times \mathrm{CH}_{3} \mathrm{MOM}\right)$.

To a solution containing the above-mentioned methyl ester derivative (540 $\mathrm{mg}, 1.71 \mathrm{mmol})$ in $\mathrm{THF}(15 \mathrm{ml})$ at $0^{\circ} \mathrm{C}$ (ice-bath), a solution of $\mathrm{LiOH} \cdot \mathrm{H}_{2} \mathrm{O}(220 \mathrm{mg}, 5.12 \mathrm{mmol})$ in water $(10 \mathrm{ml})$ was added, and the mixture was stirred at room temperature overnight. Then $1 \mathrm{~N}$ hydrochloric acid aqueous solution was added to reach $\mathrm{pH}=2$, and volatiles were evaporated to dryness. The residue was dissolved in ethyl acetate $(20 \mathrm{ml})$ and washed with $\mathrm{H}_{2} \mathrm{O}(3 \times 20 \mathrm{ml})$. The organic phase was dried over anhydrous $\mathrm{Na}_{2} \mathrm{SO}_{4}$, filtered, and evaporated to dryness to give $488.7 \mathrm{mg}$ (95\%) of the title compound that was directly used for the next step. Characterization of this compound is consistent with those found in the literature (Gažák et al., 2011).

General Coupling Procedure for the Synthesis of the MOMProtected Intermediates 8-12. A solution of the MOM-protected gallic acid 7 (Gažák et al., 2011) (2.2 eq), HATU (2.2 eq), and dimethylaminopyridine (DMAP) (1.25 eq) in dry $\mathrm{CH}_{2} \mathrm{Cl}_{2}(10 \mathrm{ml})$ was stirred at room temperature for 15 minutes and then added to a second solution, also stirred for 15 minutes, containing the corresponding serinol derivate ( $1 \mathrm{eq})$ and $\operatorname{DMAP}(1.25 \mathrm{eq})$ in dry $\mathrm{CH}_{2} \mathrm{Cl}_{2}(10 \mathrm{ml})$. The mixture was stirred at room temperature overnight and then evaporated to dryness. The residue was dissolved in ethyl acetate $(20 \mathrm{ml})$ and washed successively with saturated solutions of citric acid $(3 \times$ $20 \mathrm{ml}), \mathrm{NaHCO}_{3}(3 \times 20 \mathrm{ml})$, and $\mathrm{NaCl}(1 \times 20 \mathrm{ml})$. The organic layer was dried over anhydrous $\mathrm{Na}_{2} \mathrm{SO}_{4}$, filtered, and concentrated under vacuum. The residue was then purified by flash column chromatography using hexane/EtOAc (10:1 to 1:1) as the eluent.

Bis-O-[3,4,5-Tris(Methoxymethoxy)Galloy]-N-Propanoyl Serinol (8). Following the general procedure, $2(50 \mathrm{mg}, 0.34 \mathrm{mmol})$ was treated with 7 (Gažák et al., 2011) (226.24 mg, $0.74 \mathrm{mmol})$, HATU (284.4 mg, $0.74 \mathrm{mmol}$ ), and DMAP (226.0 mg, $1.84 \mathrm{mmol})$ to afford $366 \mathrm{mg}(75 \%)$ of the title compound as an amorphous white solid. ${ }^{1} \mathrm{H}-\mathrm{NMR}\left(300 \mathrm{MHz}, \mathrm{CD}_{3} \mathrm{Cl}\right) \delta 7.52(\mathrm{~s}, 4 \mathrm{H}, \mathrm{Ph}), 6.03(\mathrm{~d}, J=8.30 \mathrm{~Hz}, 1 \mathrm{H}$, $\mathrm{NH}), 5.24\left(\mathrm{~s}, 8 \mathrm{H}, 4 \times \mathrm{CH}_{2} \mathrm{MOM}\right), 5.22\left(\mathrm{~s}, 4 \mathrm{H}, 2 \times \mathrm{CH}_{2} \mathrm{MOM}\right), 4.72$ $(\mathrm{m}, 1 \mathrm{H}, \mathrm{CH}), 4.46\left(\mathrm{~m}, 4 \mathrm{H}, 2 \times \mathrm{CH}_{2}\right), 3.61\left(\mathrm{~s}, 6 \mathrm{H}, 2 \times \mathrm{CH}_{3} \mathrm{MOM}\right), 3.50$ (s, $\left.12 \mathrm{H}, 4 \times \mathrm{CH}_{3} \mathrm{MOM}\right), 2.23\left(\mathrm{q}, J=7.56 \mathrm{~Hz}, 2 \mathrm{H}, \mathrm{CH}_{2}\right), 1.13(\mathrm{t}, J=$ $\left.7.43 \mathrm{~Hz}, 3 \mathrm{H}, \mathrm{CH}_{3}\right)$.

Bis-O-[3,4,5-Tris(Methoxymethoxy)Galloy]-N-Dodecanoyl Serinol (9). Following the general procedure, 3 (50 mg, $0.18 \mathrm{mmol})$ was treated with 7 (Gažák et al., 2011) (120 mg, $0.40 \mathrm{mmol})$, HATU (152 mg, $0.40 \mathrm{mmol}$ ), and DMAP (244.34 mg, $0.44 \mathrm{mmol})$ to afford $72 \mathrm{mg}(47 \%)$ of the title compound as an amorphous white solid.
${ }^{1} \mathrm{H}-\mathrm{NMR}\left(300 \mathrm{MHz}, \mathrm{CD}_{3} \mathrm{Cl}\right) \delta 7.57(\mathrm{~s}, 4 \mathrm{H}, \mathrm{Ph}), 6.01(\mathrm{~d}, J=8.30 \mathrm{~Hz}, 1 \mathrm{H}$, $\mathrm{NH}), 5.24\left(\mathrm{~s}, 8 \mathrm{H}, 4 \times \mathrm{CH}_{2} \mathrm{MOM}\right), 5.22\left(\mathrm{~s}, 4 \mathrm{H}, 2 \times \mathrm{CH}_{2} \mathrm{MOM}\right), 4.73$ (m, 1H, CH), $4.45\left(\mathrm{~m}, 4 \mathrm{H}, 2 \times \mathrm{CH}_{2}\right), 3.61\left(\mathrm{~s}, 6 \mathrm{H}, 2 \times \mathrm{CH}_{3} \mathrm{MOM}\right), 3.51$ (s, $\left.12 \mathrm{H}, 4 \times \mathrm{CH}_{3} \mathrm{MOM}\right), 2.18\left(\mathrm{t}, J=7.28 \mathrm{~Hz}, 2 \mathrm{H}, \mathrm{CH}_{2}\right), 1.59(\mathrm{~m}, 2 \mathrm{H}$, $\left.\mathrm{CH}_{2}\right), 1.25\left(\mathrm{~m}, 16 \mathrm{H}, 8 \times \mathrm{CH}_{2}\right), 0.87\left(\mathrm{t}, J=6.92 \mathrm{~Hz}, 3 \mathrm{H}, \mathrm{CH}_{3}\right)$.

Bis-O-[3,4,5-Tris(Methoxymethoxy)Galloy]-N-Octadecanoyl Serinol (10). Following the general procedure, 4 (50 mg, $0.14 \mathrm{mmol})$ was treated with 7 (Gažák et al., 2011) (90.68 mg, $0.30 \mathrm{mmol}$ ), HATU (114 $\mathrm{mg}, 0.30 \mathrm{mmol}$ ), and DMAP $(42.8 \mathrm{mg}, 0.34 \mathrm{mmol})$ to afford $100.48 \mathrm{mg}(75 \%)$ of the title compound as an amorphous white solid. ${ }^{1} \mathrm{H}-\mathrm{NMR}\left(300 \mathrm{MHz}, \mathrm{CD}_{3} \mathrm{Cl}\right) \delta 7.56(\mathrm{~s}, 4 \mathrm{H}, \mathrm{Ph}), 6.02(\mathrm{~d}, J=8.30 \mathrm{~Hz}, 1 \mathrm{H}$, $\mathrm{NH}), 5.24\left(\mathrm{~s}, 8 \mathrm{H}, 4 \times \mathrm{CH}_{2} \mathrm{MOM}\right), 5.22\left(\mathrm{~s}, 4 \mathrm{H}, 2 \times \mathrm{CH}_{2} \mathrm{MOM}\right), 4.72$ (m, $1 \mathrm{H}, \mathrm{CH}), 4.44\left(\mathrm{~m}, 4 \mathrm{H}, 2 \times \mathrm{CH}_{2} \mathrm{O}\right), 3.60\left(\mathrm{~s}, 6 \mathrm{H}, 2 \times \mathrm{CH}_{3} \mathrm{MOM}\right), 3.50$ $\left(\mathrm{s}, 12 \mathrm{H}, 4 \times \mathrm{CH}_{3} \mathrm{MOM}\right), 2.05\left(\mathrm{t}, J=7.2 \mathrm{~Hz}, 2 \mathrm{H}, \mathrm{CH}_{2} \mathrm{CO}\right), 1.44$ $\left(\mathrm{m}, 2 \mathrm{H}, \mathrm{CH}_{2}\right), 1.17\left(\mathrm{~m}, 28 \mathrm{H}, 14 \times \mathrm{CH}_{2}\right), 0.81\left(\mathrm{t}, J=6.92 \mathrm{~Hz}, 3 \mathrm{H}, \mathrm{CH}_{3}\right)$.

Bis-O-[3,4,5-Tris(Methoxymethoxy)Galloy]- $N$-Oleoyl Serinol (11). Following the general procedure, $5(50 \mathrm{mg}, 0.14 \mathrm{mmol})$ was treated with 7 (Gažák et al., 2011) (90.68 mg, $0.30 \mathrm{mmol}$ ), HATU (114 $\mathrm{mg}, 0.30 \mathrm{mmol}$ ), and DMAP (42.8 $\mathrm{mg}, 0.34 \mathrm{mmol})$ to afford $106 \mathrm{mg}$ $(81 \%)$ of the title compound as an amorphous white solid. ${ }^{1} \mathrm{H}-\mathrm{NMR}$ $\left(300 \mathrm{MHz}, \mathrm{CD}_{3} \mathrm{Cl}\right) \delta 7.52(\mathrm{~s}, 4 \mathrm{H}, \mathrm{Ph}), 6.02(\mathrm{~d}, J=8.20 \mathrm{~Hz}, 1 \mathrm{H}, \mathrm{NH}), 5.33$ $(\mathrm{m}, 2 \mathrm{H}, \mathrm{CH}=\mathrm{CH}), 5.24\left(\mathrm{~s}, 8 \mathrm{H}, 4 \times \mathrm{CH}_{2} \mathrm{MOM}\right), 5.22(\mathrm{~s}, 4 \mathrm{H}, 2 \times$ $\left.\mathrm{CH}_{2} \mathrm{MOM}\right), 4.73(\mathrm{~m}, 1 \mathrm{H}, \mathrm{CH}), 4.46\left(\mathrm{~m}, 4 \mathrm{H}, 2 \times \mathrm{CH}_{2}\right), 3.61(\mathrm{~s}, 6 \mathrm{H}, 2 \times$ $\mathrm{CH}_{3} \mathrm{MOM}$ ), 3.51 (s, $\left.12 \mathrm{H}, 4 \times \mathrm{CH}_{3} \mathrm{MOM}\right), 2.19\left(\mathrm{t}, J=8.55 \mathrm{~Hz}, 2 \mathrm{H}, \mathrm{CH}_{2}\right)$, $1.99\left(\mathrm{~m}, 2 \mathrm{H}, \mathrm{CH}_{2}\right), 1.59\left(\mathrm{~m}, 4 \mathrm{H}, 2 \times \mathrm{CH}_{2}\right), 1.27\left(\mathrm{~m}, 20 \mathrm{H}, 10 \times \mathrm{CH}_{2}\right), 0.88$ (t, $J=7.44 \mathrm{~Hz}, 3 \mathrm{H}, \mathrm{CH}_{3}$ ).

Bis-O-[3,4,5-Tris(Methoxymethoxy)Galloy]- $N$-Linoleoyl Serinol (12). Following the general procedure, $6(50 \mathrm{mg}, 0.14 \mathrm{mmol})$ was treated with 7 (Gažák et al., 2011) (90.68 mg, $0.30 \mathrm{mmol}$ ), HATU (114 $\mathrm{mg}, 0.30 \mathrm{mmol}$ ), and DMAP (42.8 $\mathrm{mg}, 0.34 \mathrm{mmol})$ to afford $98.72 \mathrm{mg}(76 \%)$ of the title compound as an amorphous white solid. ${ }^{1} \mathrm{H}-\mathrm{NMR}\left(300 \mathrm{MHz}, \mathrm{CD}_{3} \mathrm{Cl}\right) \delta 7.52(\mathrm{~s}, 4 \mathrm{H}, \mathrm{Ph}), 6.00(\mathrm{~d}, J=8.20 \mathrm{~Hz}, 1 \mathrm{H}$, $\mathrm{NH}), 5.34(\mathrm{~m}, 4 \mathrm{H}, 2 \times \mathrm{CH}=\mathrm{CH}), 5.24\left(\mathrm{~s}, 8 \mathrm{H}, 4 \times \mathrm{CH}_{2} \mathrm{MOM}\right), 5.22$ $\left(\mathrm{s}, 4 \mathrm{H}, 2 \times \mathrm{CH}_{2} \mathrm{MOM}\right), 4.73(\mathrm{~m}, 1 \mathrm{H}, \mathrm{CH}), 4.46\left(\mathrm{~m}, 4 \mathrm{H}, 2 \times \mathrm{CH}_{2}\right), 3.61$ (s, 6H, $\left.2 \times \mathrm{CH}_{3} \mathrm{MOM}\right), 3.50\left(\mathrm{~s}, 12 \mathrm{H}, 4 \times \mathrm{CH}_{3} \mathrm{MOM}\right), 2.76(\mathrm{t}, J=$ $\left.7.70 \mathrm{~Hz}, 2 \mathrm{H}, \mathrm{CH}_{2}\right), 2.19\left(\mathrm{~m}, 2 \mathrm{H}, \mathrm{CH}_{2}\right), 2.03\left(\mathrm{~m}, 4 \mathrm{H}, 2 \times \mathrm{CH}_{2}\right), 1.29$ $\left(\mathrm{m}, 16 \mathrm{H}, 8 \times \mathrm{CH}_{2}\right), 0.88\left(\mathrm{t}, J=8.01 \mathrm{~Hz}, 3 \mathrm{H}, \mathrm{CH}_{3}\right)$.

Synthesis of the Deprotected Serinol Derivatives 13-17. A solution of the corresponding MOM-protected derivative in methanol was treated with aqueous hydrochloric acid (37\%). The solution was stirred at room temperature overnight and then evaporated to dryness. The residue was coevaporated several times with dichloromethane and then purified by triturating with cold diethyl ether. The precipitate that appeared was filtered and washed repeatedly with gentle quantities of cold dichloromethane and diethyl ether to afford the corresponding unprotected derivative as a white solid.

Bis-O-Galloy- $\boldsymbol{N}$-Propanoyl Serinol (13). Following the general procedure, $8(73 \mathrm{mg})$ was dissolved in methanol $(1.5 \mathrm{ml})$ and treated with aqueous $\mathrm{HCl} 37 \%(0.08 \mathrm{ml})$ to give $41.4 \mathrm{mg}(90 \%)$ of the title compound as a white amorphous solid. ${ }^{1} \mathrm{H}-\mathrm{NMR}\left(400 \mathrm{MHz}, \mathrm{DMSO}-d_{6}\right)$ $\delta 8.08(\mathrm{~d}, J=8.10 \mathrm{~Hz}, \mathrm{NH}), 6.96(\mathrm{~s}, 4 \mathrm{H}, \mathrm{Ph}), 4.39(\mathrm{~m}, 1 \mathrm{H}, \mathrm{CH}), 4.23(\mathrm{~m}$, $\left.4 \mathrm{H}, 2 \times \mathrm{CH}_{2} \mathrm{O}\right), 2.09\left(\mathrm{q}, J=7.60 \mathrm{~Hz}, 2 \mathrm{H}, \mathrm{CH}_{2}\right), 0.97(\mathrm{t}, J=7.60 \mathrm{~Hz}, 3 \mathrm{H}$, $\left.\mathrm{CH}_{3}\right) .{ }^{13} \mathrm{C}-\mathrm{NMR}\left(75 \mathrm{MHz}\right.$, DMSO- $\left.d_{6}\right) \delta 173.35,165.75,145.61,138.66$, $119.19,108.86,62.98,47.08,38.31,28.56,10.01 . \mathrm{MS}\left(\mathrm{ES}^{+}\right): \mathrm{m} / \mathrm{z} 452.1$ $(\mathrm{M}+\mathrm{H})^{+}$Anal. $\mathrm{C}_{20} \mathrm{H}_{21} \mathrm{NO}_{11}(\mathrm{C}, \mathrm{H}, \mathrm{N}, \mathrm{O})$.

Bis-O-Galloy- $\boldsymbol{N}$-Dodecanoyl Serinol (14). Following the general procedure, $9(79 \mathrm{mg})$ was dissolved in methanol $(1.5 \mathrm{ml})$ and treated with aqueous $\mathrm{HCl} 37 \%(0.08 \mathrm{ml})$ to give $51.3 \mathrm{mg}(95 \%)$ of the title compound as a white amorphous solid. ${ }^{1} \mathrm{H}-\mathrm{NMR}(400 \mathrm{MHz}$, DMSO- $\left.d_{6}\right) \delta 9.25(\mathrm{~s}, 6 \mathrm{H}, \mathrm{OH}), 8.08(\mathrm{~d}, J=8.10 \mathrm{~Hz}, \mathrm{NH}), 6.96(\mathrm{~s}, 4 \mathrm{H}$, $\mathrm{Ph}), 4.39(\mathrm{~m}, 1 \mathrm{H}, \mathrm{CH}), 4.23\left(\mathrm{~m}, 4 \mathrm{H}, 2 \times \mathrm{CH}_{2} \mathrm{O}\right), 2.09(\mathrm{q}, J=7.60 \mathrm{~Hz}, 2 \mathrm{H}$, $\left.\mathrm{CH}_{2}\right), 1.50\left(\mathrm{~m}, 2 \mathrm{H}, \mathrm{CH}_{2}\right), 1.17\left(\mathrm{~m}, 16 \mathrm{H}, 8 \times \mathrm{CH}_{2}\right), 0.97(\mathrm{t}, J=7.60 \mathrm{~Hz}$, $\left.3 \mathrm{H}, \mathrm{CH}_{3}\right) .{ }^{13} \mathrm{C}-\mathrm{NMR}\left(75 \mathrm{MHz}, \mathrm{DMSO}-d_{6}\right) \delta 165.05,144.08,137.99$, 108.12, 62.33, 30.66, 28.39, 28.35, 28.24, 28.15, 28.09, 21.47, 13.33. MS $\left(\mathrm{ES}^{+}\right): \mathrm{m} / \mathrm{z} 578.2(\mathrm{M}+\mathrm{H})^{+}$. Anal. $\mathrm{C}_{29} \mathrm{H}_{39} \mathrm{NO}_{11}(\mathrm{C}, \mathrm{H}, \mathrm{N}, \mathrm{O})$.

Bis-O-Galloy- $\boldsymbol{N}$-Octadecanoyl Serinol (15). Following the general procedure, $10(70 \mathrm{mg})$ was dissolved in methanol $(1.5 \mathrm{ml})$ and treated with aqueous $\mathrm{HCl} 37 \%(0.08 \mathrm{ml})$ to give $39 \mathrm{mg}(92 \%)$ of the title 
compound as a white amorphous solid. ${ }^{1} \mathrm{H}-\mathrm{NMR}\left(400 \mathrm{MHz}, \mathrm{DMSO}-d_{6}\right)$ $\delta 9.13(\mathrm{~s}, 6 \mathrm{H}, \mathrm{OH}), 8.07(\mathrm{~d}, J=6.94 \mathrm{~Hz}, \mathrm{NH}), 6.93(\mathrm{~s}, 4 \mathrm{H}, \mathrm{Ph}), 4.38$ $(\mathrm{m}, 1 \mathrm{H}, \mathrm{CH}), 4.21\left(\mathrm{~m}, 4 \mathrm{H}, 2 \times \mathrm{CH}_{2} \mathrm{O}\right), 2.05\left(\mathrm{t}, J=7.20 \mathrm{~Hz}, 2 \mathrm{H}, \mathrm{CH}_{2} \mathrm{CO}\right)$, $1.43\left(\mathrm{~m}, 2 \mathrm{H}, \mathrm{CH}_{2}\right), 1.17\left(\mathrm{~m}, 28 \mathrm{H}, 14 \times \mathrm{CH}_{2}\right), 0.81(\mathrm{t}, J=7.08 \mathrm{~Hz}, 3 \mathrm{H}$, $\left.\mathrm{CH}_{3}\right) .{ }^{13} \mathrm{C}-\mathrm{NMR}\left(75 \mathrm{MHz}, \mathrm{DMSO}-d_{6}\right) \delta 172.5,165.7,145.5,138.6$, 119.1, 108.7, 63.0, 46.9, 38.3, 35.4, 31.3, 29.1, 28.9, 28.8, 28.7, 28.6, 25.3, 22.1, 14.0. MS $\left(\mathrm{ES}^{+}\right): \mathrm{m} / \mathrm{z} 662.3(\mathrm{M}+\mathrm{H})^{+}$. Anal. $\mathrm{C}_{35} \mathrm{H}_{51} \mathrm{NO}_{11}$ (C, H, N, O).

Bis-O-Galloy- $\boldsymbol{N}$-Oleoyl Serinol (16). Following the general procedure, $11(71.4 \mathrm{mg})$ was dissolved in methanol $(1.5 \mathrm{ml})$ and treated with aqueous $\mathrm{HCl} 37 \%(0.08 \mathrm{ml})$ to give $45.9 \mathrm{mg}(90 \%)$ of the title compound as a white amorphous solid. ${ }^{1} \mathrm{H}-\mathrm{NMR}\left(400 \mathrm{MHz}, \mathrm{DMSO}-d_{6}\right)$ $\delta 9.23(\mathrm{~s}, 4 \mathrm{H}, \mathrm{OH}), 8.96(\mathrm{~s}, 2 \mathrm{H}, \mathrm{OH}), 8.07(\mathrm{~d}, J=6.94 \mathrm{~Hz}, \mathrm{NH}), 6.95(\mathrm{~s}$, $4 \mathrm{H}, \mathrm{Ph}), 5.28(\mathrm{~m}, 2 \mathrm{H}, \mathrm{CH}=\mathrm{CH}), 4.40(\mathrm{~m}, 1 \mathrm{H}, \mathrm{CH}), 4.22(\mathrm{~m}, 4 \mathrm{H}, 2 \times$ $\left.\mathrm{CH}_{2}\right), 2.06\left(\mathrm{t}, J=7.20 \mathrm{~Hz}, 2 \mathrm{H}, \mathrm{CH}_{2}\right), 1.92\left(\mathrm{~m}, 4 \mathrm{H}, 2 \mathrm{CH}_{2}\right), 1.44(\mathrm{~m}, 2 \mathrm{H}$, $\left.\mathrm{CH}_{2}\right), 1.20\left(\mathrm{~m}, 20 \mathrm{H}, 10 \times \mathrm{CH}_{2}\right), 0.82\left(\mathrm{t}, J=7.08 \mathrm{~Hz}, 3 \mathrm{H}, \mathrm{CH}_{3}\right)$. ${ }^{13} \mathrm{C}-\mathrm{NMR}\left(75 \mathrm{MHz}, \mathrm{DMSO}-d_{6}\right) \delta$ 172.4. 165.7, 145.5, 138.6, 129.7, $129.6,119.1,108.7,63.0,46.9,35.4,31.3,29.1,28.8,28.7,28.6,28.5$, 26.6, 25.3, 22.1, 14.0. MS $\left(\mathrm{ES}^{+}\right): \mathrm{m} / \mathrm{z} 660.3(\mathrm{M}+\mathrm{H})^{+}$. Anal. $\mathrm{C}_{35} \mathrm{H}_{49} \mathrm{NO}_{11}$ (C, H, N, O).

Bis-O-Galloy- $\boldsymbol{N}$-Linoleoyl Serinol (17). Following the general procedure, $12(61.5 \mathrm{mg})$ was dissolved in methanol $(1.5 \mathrm{ml})$ and treated with $\mathrm{HCl} 37 \%(0.08 \mathrm{ml})$ to give $42.5 \mathrm{mg}(97 \%)$ of the title compound as a white amorphous solid. ${ }^{1} \mathrm{H}-\mathrm{NMR}\left(400 \mathrm{MHz}, \mathrm{DMSO}-d_{6}\right) \delta 8.07(\mathrm{~d}, J=$ $6.94 \mathrm{~Hz}, \mathrm{NH}), 6.93(\mathrm{~s}, 4 \mathrm{H}, \mathrm{Ph}), 5.26(\mathrm{~m}, 4 \mathrm{H}, 2 \times \mathrm{CH}=\mathrm{CH}), 4.38(\mathrm{~m}, 1 \mathrm{H}$, $\mathrm{CH}), 4.20\left(\mathrm{~m}, 4 \mathrm{H}, 2 \times \mathrm{CH}_{2}\right), 2.71\left(\mathrm{~m}, 2 \mathrm{H}, \mathrm{CH}_{2}\right), 2.05(\mathrm{t}, J=7.20 \mathrm{~Hz}, 2 \mathrm{H}$, $\left.\mathrm{CH}_{2}\right), 1.90\left(\mathrm{~m}, 2 \mathrm{H}, \mathrm{CH}_{2}\right), 1.43\left(\mathrm{~m}, 2 \mathrm{H}, \mathrm{CH}_{2}\right), 1.19\left(\mathrm{~m}, 18 \mathrm{H}, 9 \times \mathrm{CH}_{2}\right)$, $0.80\left(\mathrm{t}, J=7.08 \mathrm{~Hz}, 3 \mathrm{H}, \mathrm{CH}_{3}\right) .{ }^{13} \mathrm{C}-\mathrm{NMR}\left(75 \mathrm{MHz}\right.$, DMSO- $\left.d_{6}\right) \delta 172.7$, 165.8, 145.6, 138.7, 129.9, 129.8, 127.9, 127.8, 119.2, 108.9, 63.1, 47.1, $35.5,31.0,29.2,28.8,28.7,28.6,26.7,25.5,25.3,22.1,14.1 . \mathrm{MS}\left(\mathrm{ES}^{+}\right)$: $\mathrm{m} / \mathrm{z} 658.3(\mathrm{M}+\mathrm{H})^{+}$. Anal. $\mathrm{C}_{35} \mathrm{H}_{47} \mathrm{NO}_{11}(\mathrm{C}, \mathrm{H}, \mathrm{N}, \mathrm{O})$.

Bis-O-[2,3-Bis(Benzyloxy)Benzoyl]- $N$-Oleoyl Serinol (19). A solution of the Bn-protected benzoic acid $\mathbf{1 8}$ (Belin et al., 2003) (166 mg, $0.49 \mathrm{mmol})$, HATU (214 mg, $0.56 \mathrm{mmol})$, and DMAP (41.5, $0.33 \mathrm{mmol})$ in dry $\mathrm{CH}_{2} \mathrm{Cl}_{2}(7.5 \mathrm{ml})$ was stirred at room temperature for 15 minutes and then added to a second solution, also stirred for 15 minutes, containing the $N$-oleoyl serinol derivate $5(80 \mathrm{mg}$, $0.22 \mathrm{mmol})$ and $\operatorname{DMAP}(41.5,0.33 \mathrm{mmol})$ in dry $\mathrm{CH}_{2} \mathrm{Cl}_{2}(7.5 \mathrm{ml})$. The mixture was stirred at room temperature overnight and then evaporated to dryness. The residue was dissolved in ethyl acetate $(20 \mathrm{ml})$ and washed successively with saturated solutions of citric acid $(3 \times$ $20 \mathrm{ml}), \mathrm{NaHCO}_{3}(3 \times 20 \mathrm{ml})$, and $\mathrm{NaCl}(1 \times 20 \mathrm{ml})$. The organic layer was dried over anhydrous $\mathrm{Na}_{2} \mathrm{SO}_{4}$, filtered, and concentrated under vacuum. The residue was then purified by flash column chromatography using hexane/EtOAc (10:1 to 1:1) as the eluent to afford $174 \mathrm{mg}$ $(77 \%)$ of the title compound as a white amorphous solid. ${ }^{1} \mathrm{H}-\mathrm{NMR}$ $\left(400 \mathrm{MHz}, \mathrm{CDCl}_{3}\right) \delta 7.42-7.06(\mathrm{~m}, 26 \mathrm{H}), 5.77(\mathrm{~d}, 1 \mathrm{H}, \mathrm{NH}), 5.33(\mathrm{~m}, 2 \mathrm{H}$, $\mathrm{CH}=\mathrm{CH}), 5.13\left(\mathrm{~m}, 8 \mathrm{H}, 4 \times \mathrm{CH}_{2}\right), 4.56(\mathrm{~m}, 1 \mathrm{H}, \mathrm{CH}), 4.39\left(\mathrm{~m}, 2 \mathrm{H}, \mathrm{CH}_{2}\right)$, $4.28\left(\mathrm{~m}, 2 \mathrm{H}, \mathrm{CH}_{2}\right), 2.06\left(\mathrm{~m}, 4 \mathrm{H}, 2 \mathrm{CH}_{2}\right), 1.95\left(\mathrm{~m}, 2 \mathrm{H}, \mathrm{CH}_{2}\right), 1.20(\mathrm{~m}, 20 \mathrm{H}$, $\left.10 \times \mathrm{CH}_{2}\right), 0.85\left(\mathrm{t}, J=7.08 \mathrm{~Hz}, 3 \mathrm{H}, \mathrm{CH}_{3}\right){ }^{13} \mathrm{C}-\mathrm{NMR}\left(75 \mathrm{MHz}, \mathrm{CDCl}_{3}\right) \delta$ $173.1,166.0,152.8,148.5,137.4,136.6,130.1$, 129.9, 128.7, 128.6, $128.5,128.3,128.2,127.7,126.3,124.2,123.1,118.3,75.8,71.4,63.7$, 47.4, 36.5, 32.0, 29.9, 29.7, 29.5, 29.4, 29.3, 27.4, 25.6, 22.8, 14.3.

Bis-O-(2,3-Dihydroxybenzoyl)- $N$-Octadecanoyl Serinol (20). A solution of $19(150 \mathrm{mg}, 0.152 \mathrm{mmol})$ in THF/MeOH (1:1) containing 30\% wt $\%$ of $\mathrm{Pd} / \mathrm{C}(10 \%)$ was hydrogenated at $30^{\circ} \mathrm{C}$ overnight under atmospheric pressure using a reaction balloon filled with hydrogen gas and a glass flask as the reaction vessel. The $\mathrm{Pd} / \mathrm{C}$ was filtered through Whatman filter paper 42 , and the solvent was removed under reduced pressure to give a crude product, which was then purified by triturating with cold diethyl ether and dichloromethane to afford $84 \mathrm{mg}(87 \%)$ of the title compound as a white amorphous solid. ${ }^{1} \mathrm{H}-\mathrm{NMR}\left(400 \mathrm{MHz}, \mathrm{DMSO}-d_{6}\right) \delta$ $10.27(\mathrm{~s}, 2 \mathrm{H}, \mathrm{OH}), 9.38(\mathrm{~s}, 2 \mathrm{H}, \mathrm{OH}), 8.12(\mathrm{~d}, J=8.20 \mathrm{~Hz}, \mathrm{NH}), 7.26(\mathrm{dd}, J=$ $8.10 \mathrm{~Hz}, 2 \mathrm{H}, J=0.60 \mathrm{~Hz}, 2 \mathrm{H}, \mathrm{Ph}), 7.00$ (dd, $J=7.80 \mathrm{~Hz}, J=0.60 \mathrm{~Hz}, 2 \mathrm{H}$, $\mathrm{Ph}), 6.70(\mathrm{t}, J=7.90 \mathrm{~Hz}, 2 \mathrm{H}, \mathrm{Ph}), 4.54(\mathrm{~m}, 1 \mathrm{H}, \mathrm{CH}), 4.38\left(\mathrm{~m}, 4 \mathrm{H}, 2 \times \mathrm{CH}_{2}\right)$, $2.06\left(\mathrm{t}, J=7.20 \mathrm{~Hz}, 2 \mathrm{H}, \mathrm{CH}_{2}\right), 1.43\left(\mathrm{~m}, 2 \mathrm{H}, \mathrm{CH}_{2}\right), 1.16\left(\mathrm{~m}, 28 \mathrm{H}, 14 \times \mathrm{CH}_{2}\right)$, $0.82\left(\mathrm{t}, J=6.60 \mathrm{~Hz}, 3 \mathrm{H}, \mathrm{CH}_{3}\right) .{ }^{13} \mathrm{C}-\mathrm{NMR}\left(75 \mathrm{MHz}, \mathrm{DMSO}-d_{6}\right) \delta 172.6$, 169.2, 149.5, 146.1, 120.8, 119.9, 118.8, 112.9, 64.0, 46.4, 35.4, 31.3, 30.4,
29.1, 29.0, 28.9, 28.8, 28.7, 28.5, 25.3, 22.1, 14.0. MS $\left(\mathrm{ES}^{+}\right): \mathrm{m} / \mathrm{z} 630.4(\mathrm{M}+$ $\mathrm{H})^{+}$. Anal. $\mathrm{C}_{35} \mathrm{H}_{51} \mathrm{NO}_{9}(\mathrm{C}, \mathrm{H}, \mathrm{N}, \mathrm{O})$.

$N, N$-bis[3,4,5-Tris(Benzyloxy)Benzoyloxyethyl]Oleoylamide (23). A solution of the benzyl-protected gallic acid 21 (Rivero-Buceta et al., 2015b) (263 mg, $0.60 \mathrm{mmol})$, HATU (258 mg, $0.68 \mathrm{mmol})$, and DMAP (52.5 mg, $0.40 \mathrm{mmol})$ in dry $\mathrm{CH}_{2} \mathrm{Cl}_{2}(10 \mathrm{ml})$ was stirred at room temperature for 15 minutes and then added to a second solution, also stirred for 15 minutes, containing $22(100 \mathrm{mg}, 0.27 \mathrm{mmol})$ and DMAP ( $52.5 \mathrm{mg}, 0.40 \mathrm{mmol})$ in dry $\mathrm{CH}_{2} \mathrm{Cl}_{2}(10 \mathrm{ml})$. The mixture was stirred at room temperature overnight and then evaporated to dryness. The residue was dissolved in ethyl acetate $(20 \mathrm{ml})$ and washed successively with saturated solutions of citric acid $(3 \times 20 \mathrm{ml}), \mathrm{NaHCO}_{3}(3 \times 20 \mathrm{ml})$, and $\mathrm{NaCl}(1 \times 20 \mathrm{ml})$. The organic layer was dried over anhydrous $\mathrm{Na}_{2} \mathrm{SO}_{4}$, filtered, and concentrated under vacuum. The residue was then purified by flash column chromatography using hexane/EtOAc (4:1 to $1: 1)$ as the eluent to afford $216 \mathrm{mg} \mathrm{(66 \% )}$ of the title compound as an amorphous white solid. ${ }^{1} \mathrm{H}-\mathrm{NMR}\left(400 \mathrm{MHz}, \mathrm{CDCl}_{3}\right) \delta 7.27$ $(\mathrm{m}, 34 \mathrm{H}, \mathrm{Ph}), 5.24(\mathrm{~m}, 2 \mathrm{H}, \mathrm{CH}=\mathrm{CH}), 5.01\left(\mathrm{~m}, 12 \mathrm{H}, \mathrm{CH}_{2}\right), 4.36(\mathrm{~m}, 4 \mathrm{H}$, $\left.\mathrm{CH}_{2}\right), 4.36\left(\mathrm{~m}, 4 \mathrm{H}, \mathrm{CH}_{2}\right), 3.66\left(\mathrm{~m}, 4 \mathrm{H}, \mathrm{CH}_{2}\right), 2.29\left(\mathrm{~m}, 2 \mathrm{H}, \mathrm{CH}_{2}\right), 1.90$ $\left(\mathrm{m}, 4 \mathrm{H}, 2 \times \mathrm{CH}_{2}\right), 1.50\left(\mathrm{~m}, \mathrm{H}, \mathrm{CH}_{2}\right), 1.17\left(\mathrm{~m}, 20 \mathrm{H}, 10 \times \mathrm{CH}_{2}\right), 0.80(\mathrm{t}, J$ $\left.=6.60 \mathrm{~Hz}, 3 \mathrm{H}, \mathrm{CH}_{3}\right) .{ }^{13} \mathrm{C}-\mathrm{NMR}\left(75 \mathrm{MHz} \mathrm{CDCl}_{3}\right) \delta 173.3,166.1,165.9$, $152.8,152.7,136.8,136.6,130.1,129.9,128.7,128.6,128.3,128.2$, $128.1,127.7,127.6,125.0,124.5,109.2,100.5,75.3,71.4,71.3,63.0$, 62.6, 45.7, 33.4, 32.1, 29.9, 29.7, 29.6, 29.5, 29.4, 27.3, 25.5, 22.8, 14.3 .

$\boldsymbol{N}, \boldsymbol{N}$-Bis $(3,4,5$-Trihydroxybenzoyloxyethyl)Octadecanoylamide (24). A solution of 23 (200 mg, $0.164 \mathrm{mmol})$ in THF/MeOH (1:1) containing $30 \% \mathrm{wt} \%$ of $\mathrm{Pd} / \mathrm{C}(10 \%)$ was hydrogenated at $30^{\circ} \mathrm{C}$ overnight under atmospheric pressure using a reaction balloon filled with hydrogen gas and a glass flask as the reaction vessel. The $\mathrm{Pd} / \mathrm{C}$ was filtered through Whatman filter paper 42, and the solvent was removed under reduced pressure to give the crude product, which was then purified by triturating with cold diethyl ether and dichloromethane to afford $58 \mathrm{mg}$ (53\%) of the title compound as a white amorphous solid. ${ }^{1} \mathrm{H}-\mathrm{NMR}\left(300 \mathrm{MHz}, \mathrm{DMSO}-d_{6}\right) \delta 9.14(\mathrm{~s}, 3 \mathrm{H}, \mathrm{OH})$, $6.91(\mathrm{~s}, 4 \mathrm{H}, \mathrm{Ph}), 4.27\left(\mathrm{~m}, 4 \mathrm{H}, 2 \times \mathrm{CH}_{2}\right), 3.66\left(\mathrm{~m}, 4 \mathrm{H}, 2 \times \mathrm{CH}_{2}\right), 2.31$ $\left(\mathrm{t}, J=7.20 \mathrm{~Hz}, 2 \mathrm{H}, \mathrm{CH}_{2}\right), 1.41\left(\mathrm{~m}, 2 \mathrm{H}, \mathrm{CH}_{2}\right), 1.29\left(\mathrm{~m}, 28 \mathrm{H}, 14 \times \mathrm{CH}_{2}\right)$, $0.81\left(\mathrm{t}, J=6.60 \mathrm{~Hz}, 3 \mathrm{H}, \mathrm{CH}_{3}\right) .{ }^{13} \mathrm{C}-\mathrm{NMR}\left(75 \mathrm{MHz} \mathrm{CDCl}_{3}\right) \delta 172.6$, 165.8, 165.7, 145.6, 145.5, 138.7, 138.5, 119.2, 118.9, 108.6, 61.9, 61.7, $46.8,44.3,32.1,31.3,29.1,29.0,28.9,28.7,24.9,22.1,14.0 . \mathrm{MS}\left(\mathrm{ES}^{+}\right)$: $\mathrm{m} / \mathrm{z} 676.4(\mathrm{M}+\mathrm{H})^{+}$. Anal. $\mathrm{C}_{36} \mathrm{H}_{53} \mathrm{NO}_{11}(\mathrm{C}, \mathrm{H}, \mathrm{N}, \mathrm{O})$.

\section{Biologic Methods}

Stock solutions for each compound were prepared by dissolving the corresponding compound in DMSO and stored at $-20^{\circ} \mathrm{C}$ in dark.

Cell Culture. SW-480 and SW-620 colorectal cancer cell lines and CCD18-Co colonic fibroblastic colon cell line were obtained from the American Type Culture Collection (Manassas, VA) and were maintained under standard conditions of temperature $\left(37^{\circ} \mathrm{C}\right)$, humidity (95\%), and carbon dioxide (5\%). SW-620 and SW-480 colon cancer cell lines were cultured in Dulbecco's modified Eagle medium (GibcoInvitrogen, Grand Island, NY) supplemented with $10 \%$ fetal bovine serum (Gibco-Invitrogen), $2 \mathrm{mM}$ glutamine (BioWhittaker; Lonza Group, Basel, Switzerland), and $1 \%$ antibiotics-antifungal (containing $10,000 \mathrm{U} / \mathrm{ml}$ penicillin base, $10,000 \mu \mathrm{g} / \mathrm{ml}$ streptomycin base, and 25,000 ng/ml amphotericin B; Gibco-Invitrogen). CCD18-Co human colon fibroblasts (CRL-1459; American Type Culture Collection) were grown in minimum essential medium supplemented with $10 \%$ fetal bovine serum, $1 \%$ antibiotic/antimycotic, $2 \mathrm{mM}$ GlutaMAX, and $0.1 \mathrm{mM}$ nonessential amino acids (Invitrogen Life Technologies Corporation, Carlsbad, CA).

Cell Viability Assay. The antiproliferative and cytotoxicity properties of the compounds in SW-620 human colon cancer cell line were measured by 3-(4,5-dimethylthiazol-2-yl)-2,5-diphenyltetrazolium bromide (MTT) assay. SW-620 cells were seeded in 24-well plates in exponential growth phase using a density of $25 \times 10^{3}$ cells/well. After 24 hours, culture media were replaced with $500 \mu \mathrm{l}$ fresh culture media containing serial concentrations of each compound (dissolved in 
DMSO), and the number of viable cells was determined at time zero (control growth wells) using the MTT assay, as described below for treated cells. After 48 hours of treatment, treated cells were incubated during 3 hours at $37^{\circ} \mathrm{C}$ with MTT ( $50 \mu \mathrm{l} /$ well at $5 \mathrm{mg} / \mathrm{ml}$ in phosphatebuffered saline) (Sigma-Aldrich, St. Louis, MO). Over that time, MTTcontaining media were removed, and the MTT reduced to purple formazan by living cells was solubilized by adding $200 \mu \mathrm{l}$ DMSO per well. After 1 hour mixing in dark, reduced formazan product, proportional to the number of viable cells, was measured at $560 \mathrm{~nm}$ using a scanning spectrophotometer microplate reader (Biochrom Asys UVM 340 Microplate Reader; ISOGEN, De Meern, The Netherlands). The parameters $\mathrm{IC}_{50}$ (micromolars; concentration needed for $50 \%$ inhibition of cell proliferation), $\mathrm{GI}_{50}$ (micromolars; concentration needed for 50\% growth inhibition), TGI (micromolars) (concentration needed for total growth inhibition), and $\mathrm{LC}_{50}$ (micromolars; concentration needed for 50\% cell death) were calculated according to the National Institutes of Health definitions using a logistic regression.

For all compounds, at least three independent experiments with three replicates per concentration were performed.

Extracellular Flux Analysis of the Oxygen Consumption Rate and Extracellular Acidification Rate. Mitochondrial respiration and glycolytic function were analyzed with the XFe96 Cell Bioanalyzer and Cell Mito Stress Test and XF Glycolysis Stress kits, respectively (XFe96; Seahorse Biosciences, Chicopee, MA). Prior to the assay, optimal cell density and oligomycin tritation were determined.

Glycolysis and oxidative phosphorylation are the two main energyproducing pathways in the cell. Most cells possess the ability to switch between these two pathways, thereby adapting to changes in their environment. Glucose in the cell is converted to pyruvate (referred to as glycolysis) and then converted to lactate in the cytoplasm, or $\mathrm{CO}_{2}$ and water in the mitochondria. The conversion of glucose to pyruvate, and subsequently lactate, results in a net production and extrusion of protons into the extracellular medium. This results in the acidification of the medium, which is directly measured by XF6 analyzer and reported as the extracellular acidification rate (ECAR).

The assay scheme is as follows: First, cells are incubated in the glycolysis stress test medium without glucose or pyruvate and basal ECAR is monitored. The first injection is a saturating concentration of glucose $(10 \mathrm{mM})$. The cells catabolize this glucose to pyruvate through the glycolytic pathway, producing ATP, NADH, water, and protons. The extrusion of protons into the surrounding medium leads to an increase in basal ECAR. The difference between ECAR before (basal situation) and after the addition of glucose is a measurement of the rate of glycolysis (glycolysis rate).

The second injection is oligomycin, which inhibits ATP synthase. Oligomycin inhibits mitochondrial ATP production and shifts the energy production to glycolysis, with the subsequent increase in ECAR, which is monitored as the cellular maximum glycolytic capacity. The difference between glycolytic capacity and glycolysis rate defines glycolytic reserve.

The final injection is 2-deoxy-glucose, a glucose competitor, which inhibits glycolysis through competitive binding to hexokinase, the first enzyme in the glycolytic pathway. The resulting decrease in ECAR confirms that the ECAR produced and monitored in the assay is due to glycolysis.

For glycoStress assay, 20,000 cells were plated in a XFe 96-well plate and kept overnight in complete media. The next day, culture medium was changed to $0 \mathrm{mM}$ glucose, $2 \mathrm{Mm}$ glutamine in nonbuffered media to starve the cells for 1 hour. Basal ECAR was monitored (one to three measurements). Next, $10 \mathrm{mM}$ glucose was injected to determine the capacity of the cells to upregulate glycolysis from the basal situation (four to six measurements), and difference between basal ECAR and ECAR in the presence of glucose was determined to calculate glycolysis value. Next, oligomycin was added to block ATP production from mitochondrial respiration to determine the maximal glycolytic capacity. Finally, $50 \mathrm{mM}$ of 2-deoxyglucose was injected to block completely glycolytic pathway.
The Cell Mito Stress Test measures key parameters of mitochondrial respiration by measuring the oxygen consumption rate (OCR) of cells. Cell Mito Stress Test uses sequential drug injections to target different electron transport chain complexes in the mitochondria. In this way, different parameters such as basal respiration, ATP production, proton leak, maximal respiration, spare respiratory capacity, and nonmitochondrial respiration were determined. Injections are as follows: oligomycin injection to inhibit ATP synthase (complex V), cyanide-4-(trifluoromethoxy)phenylhydrazone (FCCP) injection to uncouple oxygen consumption from ATP production, and rotenone and antimycin A injection to inhibit complexes I and III, respectively.

As indicated, oligomycin inhibits ATP synthase (complex V), and the decrease in OCR following injection of oligomycin correlates to the mitochondrial respiration associated with cellular ATP production.

Carbonyl FCCP is an uncoupling agent that disrupts the mitochondrial membrane potential, and thus, electron flow through the electron transport chain is inhibited, and oxygen is maximally consumed by complex IV. The FCCP-stimulated OCR is used to calculate spare respiratory capacity, defined as the difference between maximal respiration and basal respiration. Spare respiratory capacity is a measure of the ability of the cell to respond to increased energy demand.

The third injection is a mix of rotenone, a complex I inhibitor, and antimycin A, a complex III inhibitor. This combination shuts down mitochondrial respiration and enables the calculation of nonmitochondrial respiration driven by processes outside the mitochondria. Proton leak, which is the remaining basal respiration not coupled to ATP production, can be a sign of mitochondrial damage or can be used as a mechanism to regulate the mitochondrial ATP production.

For mitostress assay, 40,000 cells were plated and kept overnight in complete media. The next day, culture medium was changed to $10 \mathrm{mM}$ glucose, $2 \mathrm{mM}$ glutamine, and $1 \mathrm{mM}$ pyruvate nonbuffered media, and cells were kept for 1 hour at $37^{\circ} \mathrm{C}$ in an incubator without $\mathrm{CO}_{2}$. Basal OCR was monitored (one to three measurements). Next, $1 \mu \mathrm{M}$ oligomycin was injected to determine the amount of oxygen dedicated to ATP production at mitochondria (three to six measurements). Then carbonyl FCCP was injected at $0.4 \mu \mathrm{M}$ to free $\mathrm{H}^{+}$gradient from the intermembrane space to the mitochondrial matrix, and so to determine the maximal respiration rate or spare respiratory capacity. Finally, antimycin A and rotenone $(0.5 \mu \mathrm{M})$ were added to completely inhibit mitochondrial respiration (seven to nine measurements).

Western Blot. Cells were lysed in Laemmli buffer, and proteins were separated by SDS-PAGE and transferred onto a nitrocellulose membrane (Bio-Rad Laboratories, Hercules, CA). The membranes were blocked using $5 \%$ nonfat dry milk in Tris-buffered saline $0.05 \%$ Tween-20. Primary antibodies were incubated overnight at $4^{\circ} \mathrm{C}$, and, upon 1-hour incubation with secondary antibodies, signal detection was performed using the Clarity Western ECL Substrate (Bio-Rad). $\beta$-actin determination or Ponceau stain was used as loading controls.

Caspase Activation Assay. SW-620 cells were plated in 96-multiwell and treated for 48 hours with $1.66 \mu \mathrm{M}$ concentration of compounds 16 and 14. Staurosporin $(1.5 \mu \mathrm{M})$ was used as a positive control. Activity of caspase 3 and caspase 7 was quantified using the Caspase-Glo 3/7 assay kit (Promega, Madison, WI), following manufacturer's instructions.

Measurement of Cellular ATP Content. Relative cellular ATP content was measured by the ATP-based assay CellTiter-Glo Luminescent Cell Viability kit (catalogue G7571; Promega) with modifications from the manufacturer's protocol. Briefly, after 48 hours of treatment with compound 16 at $\mathrm{IC}_{50}(0.83 \mu \mathrm{M})$ and $2 \times \mathrm{IC}_{50}(1.66 \mu \mathrm{M})$, SW-620 cells were plated in 96 -well clear-bottom black polystyrene plates. Ten thousand cells were plated per well. Nontreated cells were plated in a similar way. Cells were then maintained for 6 hours in complete media, without treatments, to allow cells to attach to the plate. Then an equal volume of the single one-step reagent provided by the kit was added to each well and rocked for 15 minutes at room temperature. Cellular ATP content was measured by a luminescent plate reader. 
Statistical Analysis. Dose-response curves for cell viability assays were analyzed by analysis of variance with Bonferroni and Tukey as post hoc tests. Data were presented as mean \pm S.E.M. of at least three independent experiments, each performed in triplicate. Statistical significance was defined as $* P<0.05$; $* * P<0.01$; *** $P<0.001$. The statistical analyses were performed by use of the $R$ statistical software version 2.15 (www.r-project.org).

\section{Results}

Chemical Results. For the synthesis of the proposed final compounds, the key $N$-acyl serinol derivatives (2-6) were prepared first (Scheme 1). Selective $N$-acylation of 2 -amino1,3-propanediol (serinol, $\mathbf{1}$ ) was undertaken by reaction of the commercially available serinol with the corresponding acyl chlorides in the presence of triethylamine at $-20^{\circ} \mathrm{C}$ to afford compounds 2-6 in good yields (70\%-81\%). It should be mentioned that the synthesis of these compounds was previously described in the literature following different procedures (Oette and Tschung, 1980; Bieberich et al., 2002a,b; Schulz and Robins, 2004; Osornio et al., 2012; Landau and Siegel, 2014; Merg et al., 2015). In our case, a unique procedure that implies the use of acyl chlorides and triethylamine has been followed for all of them.

Subsequently, condensation of the $N$-acyl serinol derivatives (2-6) with the MOM-protected galloyl acid[3,4,5-tris (methoxymethoxy)benzoic acid] (7) in the presence of HATU, as coupling reagent, and DMAP as base, followed by deprotection of the MOM ether intermediates 8-12 with aqueous hydrochloric acid (37\%), gave the deprotected final compounds 13-17 in good yields (Scheme 2). It should be mentioned that the MOMprotected galloyl intermediate $\mathbf{7}$ was previously synthesized by other authors (Gažák et al., 2011). In our case, a modification of the previously described procedure, which implies the use of methyl gallate as starting material, gives this compound with good overall yield (60\% for the two steps) (Scheme 2 ).

Next, compound 20, with two 2,3-dihydroxybenzoyl residues, instead of galloyl, as phenolic substituents, was prepared (Scheme 3 ). In this case, the $N$-oleynol serinol derivative 5 was treated with the benzyl-protected benzoic acid 18 (Belin et al., 2003) in the presence of HATU, as coupling reagent, and DMAP as base (Scheme 3) to give 19 (77\%). Catalytic hydrogenation of $\mathbf{1 9}$, at atmospheric pressure in the presence of $10 \% \mathrm{Pd} / \mathrm{C}$, afforded the fully deprotected derivative 20 in $87 \%$ yield (Scheme 3 ).

The same synthetic methodology was applied for the synthesis of compound 24, in which diethanolamine, instead of serinol, has been used as linker (Scheme 4). In this case, the 3,4,5-benzyl-protected galloyl 21 (Belin et al., 2003) was used
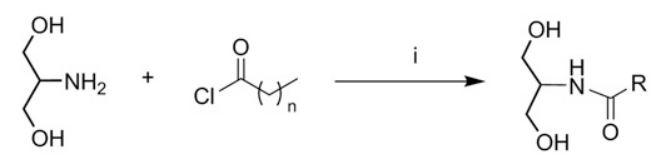

1

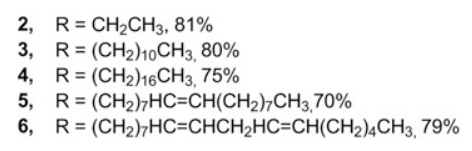

Scheme 1. Synthesis of the $N$-acyl serinol derivatives 2-6. Reagent and conditions: (i) $\mathrm{MeOH}: \mathrm{THF}(2: 1)$, Triethylamine, $-20^{\circ} \mathrm{C}$ to room temperature (rt). as the phenolic synthon. Condensation of $\mathbf{2 1}$ with the corresponding $N$-oleoyl diethanolamine derivative 22 (Bieberich et al., 2002c; Sagnella et al., 2011) afforded 23 that was submitted to catalytic hydrogenation, using $\mathrm{H}_{2} / \mathrm{Pd}-\mathrm{C}$, to give the deprotected derivative $\mathbf{2 4}$ (53\% yield).

Inhibition of Colon Cancer Cell Growth. Firstly, the antiproliferative properties of the new synthetic phenolic derivatives (13-17, 20, and 24) were tested in SW-620 human colon cancer cell line. Compound SF6, containing two galloyl moieties at both ends of a C12 $\mathrm{N}$-acyl chain, from our collection of phenolic derivatives, was included for comparative purposes (Rivero-Buceta et al., 2015a), and 4,4'-Di-O-methylellagic acid, an effective phenolic compound against colon cancer cells (Ramirez de Molina et al., 2015), was used as the reference drug. The results are shown in Table 1.

Compound SF-6 did not show any growth-inhibitory effect at the concentration tested (up to $100 \mu \mathrm{M}$ ) (Table 1), whereas compound 14, in which the two galloyl units are at the same side of the $\mathrm{C} 12 \mathrm{~N}$-acyl chain, affected human colon cell viability in the $\mu \mathrm{M}$ range. These data point to the importance for the growth-inhibitory effect of a polar head, with two galloyl subunits.

Compound 13, with one short $N$-acyl chain (C3), resulted inactive at the concentration tested. Further elongation of the length of the $N$-acyl chain led to an increase in the inhibitory activity. Thus, among the galloyl derivatives with a saturated $N$-acyl chain (13-15), the best inhibitory effect was observed for compound 15, containing a C18 alkyl substituent, followed by 14, containing a C12 alkyl chain.

In contrast, the growth-inhibitory effect observed for compound 16, with an unsaturated C18 chain, is better than that obtained for 15, with a saturated C18 chain, and also better than that of 17, with two double bonds in the acyl chain.

Compound 20 with a C-18 alkyl chain and where the phenolic substituents contain only two hydroxyl groups, was 22 -fold less active than its corresponding $\mathrm{C} 18$ analog $(\mathbf{1 5})$, with two gallic acid residues (three $\mathrm{OHs}$ on the aromatic ring).

On the basis of all of these results, it can be concluded that the cell growth inhibition depends significantly on both the size and unsaturation of the alkyl chain on the tail and the presence of galloyl groups on the head.

The importance of serinol as the linker is supported by the loss of activity of the compound $\mathbf{2 4}$, with an ethylene amine as linker, with respect to those observed for $\mathbf{1 5}$, with serinol as linker. This result indicates that simple changes in structure can result in differences in growth inhibition, serinol being the best linker.

Finally, for comparative purposes, we considered that it could be of interest to determine the growth-inhibitory effect of compound 16, the most potent, in the cancer cell line SW- 480 (Table 2). SW-480 and SW-620 are colon carcinoma cell lines from a single patient derived either from the primary tumor (SW-480) or from a lymph node metastasis (SW-620) of the same tumor. SW-620 cells are more highly tumorigenic and metastatic than SW-480 (Hewitt et al., 2000).

As it was shown in Table 2, the human colon cancer cell line SW-480 is also growth inhibited by $\mathbf{1 6}$, although to different extent than SW-620 cell line. In fact, a better inhibitory effect of 16 in SW-620 cells than in SW-480 cells $\left(\mathrm{IC}_{50}=0.83 \mu \mathrm{M}\right.$ versus $\mathrm{IC}_{50}=4.03 \mu \mathrm{M}$ ) was observed. This result can be of great therapeutic interest due to the fact that SW-620 is much more aggressive than SW-480. 


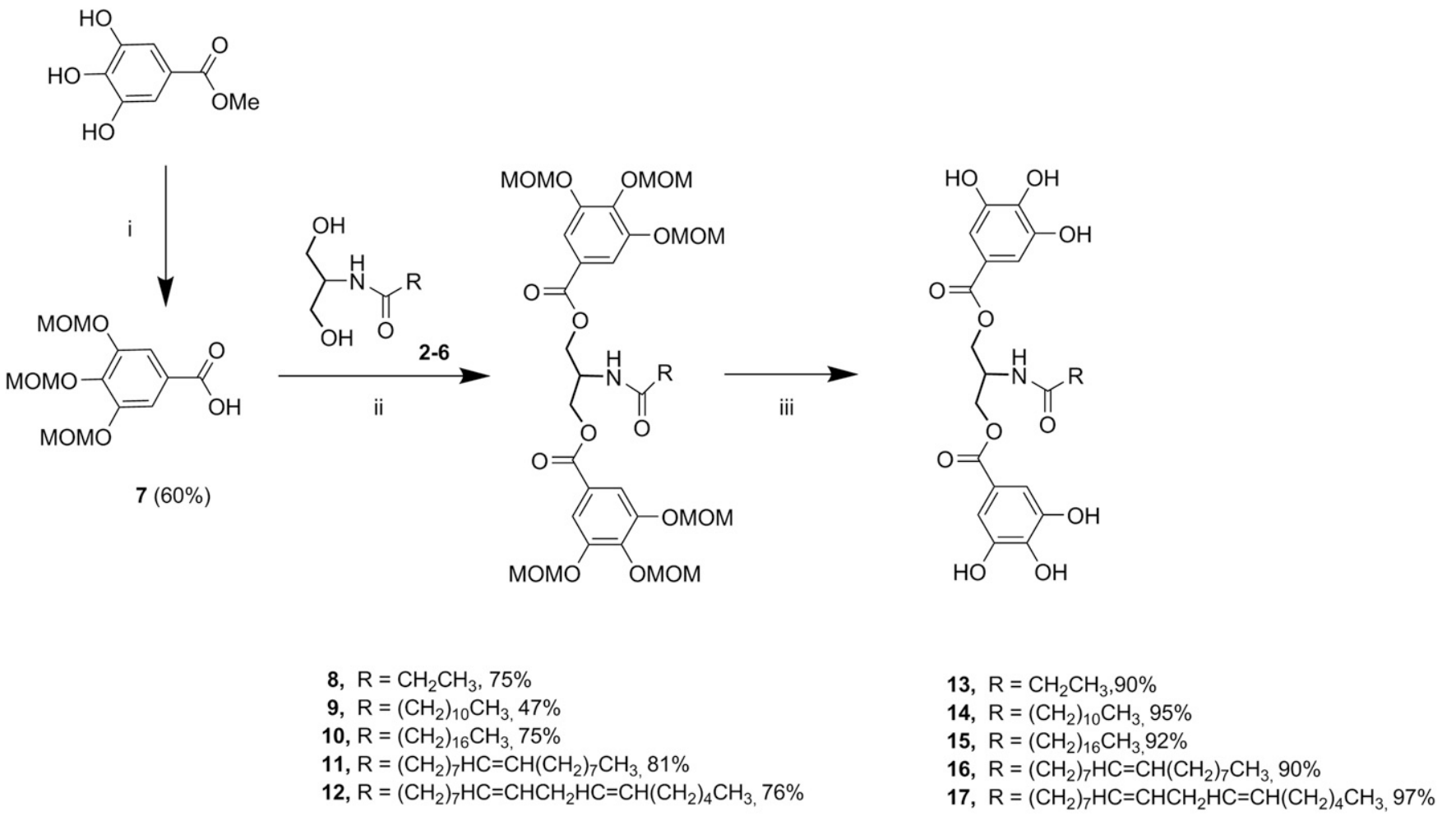

Scheme 2. Synthesis of 13-17. Reagent and conditions: (i) a) $\mathrm{MOMCl}$, b) $\mathrm{HOLi} / \mathrm{H}_{2} \mathrm{O}$ (ii) $\mathrm{HATU}$, DMAP (iii) aqueous $\mathrm{HCl}$ (37\%).

Differences in sensitivity between those two cell lines toward a particular drug have also been described in the literature (Rashmi et al., 2003). Those differences might be explained by proteomic and metabolic differences between those cell lines that required further studies.

Toxicity in Noncancer Cells. Next, toxicity in noncancer cells for compound 16, the one with the highest growthinhibitory effect, was determined. With this purpose, CCD18-Co colonic fibroblastic colon cell line was used. The $\mathrm{IC}_{50}$ for compound $\mathbf{1 6}$ in these primary epithelial colonic cells was $30 \mu \mathrm{M}$ versus $0.83 \mu \mathrm{M}$ in SW-620 colon cancer cells. This result indicates that there exists a therapeutic window for the use of compound $\mathbf{1 6}$ in colon cancer.

Cell Bioenergetic Analysis. Very recently, a rapid and easy experimental methodology has been developed to analyze the energetic metabolism of a cell (glycolysis and oxidative phosphorylation) in real time (Pike Winer and Wu, 2014;
TeSlaa and Teitell, 2014). When applied to the anticancer field, this novel methodology facilitates a better understanding of the cancer cell metabolism and may be useful to discover agents that target specific metabolic pathways.

Glycolysis, conversion of glucose to lactate with the production of two molecules of ATP, is monitored through the measurement of the extracellular acidification rate (ECAR) at the surrounding media, whereas oxidative phosphorylation is monitored through the measurement of the OCR. High levels in basal ECAR may be indicative of reliance on glycolysis to support cell proliferation. The response to an input of glucose after starvation - this is, the increase in ECAR compared with the basal situation-determines the preferential use of the glycolytic pathway versus oxidative mitochondrial pathway. In contrast, monitorization of OCR allows to determine the dependency of the oxidative metabolism by the cell. Thus, high basal OCR indicates a highly active oxidative metabolism.

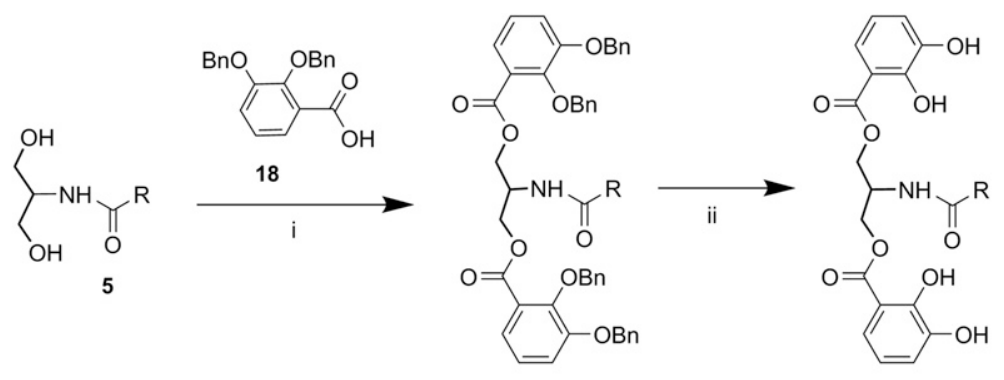

19, $\mathrm{R}=\left(\mathrm{CH}_{2}\right)_{7} \mathrm{HC}=\mathrm{CH}\left(\mathrm{CH}_{2}\right)_{7} \mathrm{CH}_{3}, 77 \%$

20, $\mathrm{R}=\left(\mathrm{CH}_{2}\right){ }_{16} \mathrm{CH}_{3}, 87 \%$

Scheme 3. Synthesis of 20. Reagent and conditions: (i) HATU, DMAP (ii) $\mathrm{H}_{2}, \mathrm{Pd} / \mathrm{C}$. 


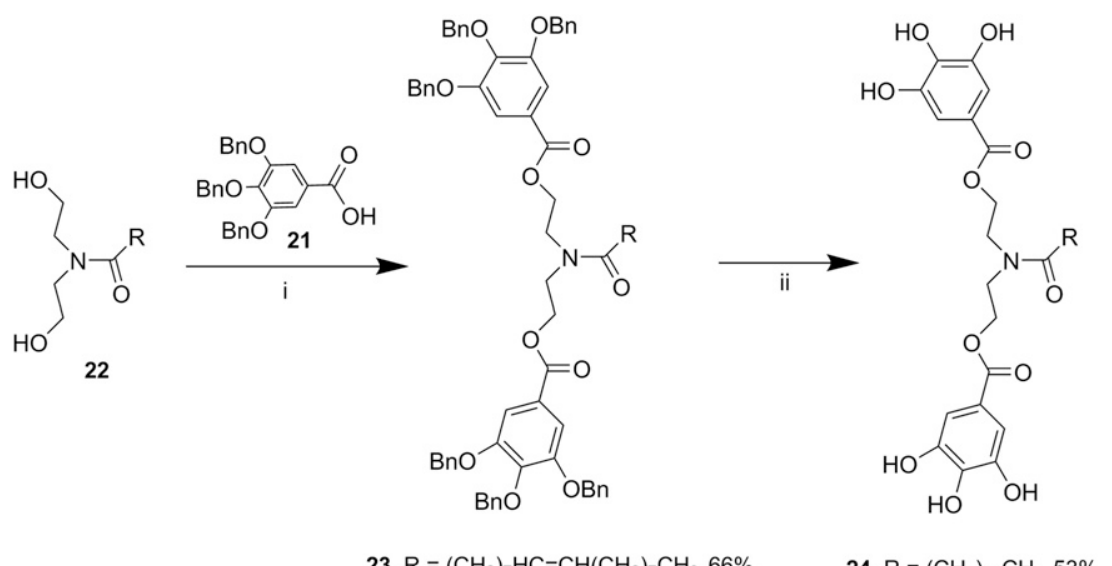

Scheme 4. Synthesis of 24. Reagent and conditions: (i) HATU, DMAP (ii) $\mathrm{H}_{2}, \mathrm{Pd} / \mathrm{C}$.

Taking all of this into consideration, we investigated the role of compound $\mathbf{1 6}\left(\mathrm{IC}_{50}: 0.83 \mu \mathrm{M}\right)$, the one with the highest growth-inhibitory effect, on cell bioenergy as a possible cause of its anticancer activity. With this aim, we monitored ECAR and OCR as readouts of glycolysis and mitochondrial respiration, respectively, using an extracellular flux bioanalyzer (XF96; Seahorse Biosciences). Compound $14\left(\mathrm{IC}_{50}: 29.5 \mu \mathrm{M}\right)$, with a moderate effect on cell viability, was also evaluated for comparative purposes.

We first tested the effect of compound $\mathbf{1 6}$ on the glycolytic activity using a glycostress standard assay (Fig. 2). SW-620 cancer cells were pretreated for 48 hours with $\mathbf{1 6}$ at two concentrations $\left(0.83 \mu \mathrm{M}=\mathrm{IC}_{50}\right.$ and $\left.1.66 \mu \mathrm{M}=2 \times \mathrm{IC}_{50}\right)$. Similarly, SW-620 cancer cells were pretreated with 14 at 0.83 and $16.66 \mu \mathrm{M}$ (onefold and 20-fold of the $\mathrm{IC}_{50}$ showed for compound 16). Then cells were resuspended and plated overnight. The next day, media was changed and cells were glucose starved for 1 hour. Basal ECAR was monitored (one to three measurements). Next, $10 \mathrm{mM}$ glucose was injected to determine the capacity of the cells to increase glycolysis from the basal situation (four to six measurements). The difference between ECAR before (basal situation) and after the addition of glucose is a measurement of the glycolytic rate (glycolysis rate). As it can be observed in Fig. 2, compound $\mathbf{1 6}$ at a concentration of $1.66 \mu \mathrm{M}$ significantly reduced glycolysis rate compared with control situation.

When oligomycin was added to inhibit mitochondrial ATP synthase and to block ATP production from mitochondrial respiration, SW-620 cells increased their glycolytic flux to maintain cell bioenergetic homeostasis. This increase in the glycolytic flux in response to a deficiency in mitochondrial ATP production is known as glycolytic capacity. As shown in Fig. 2, the glycolytic capacity was significantly diminished in the presence of a $2 \times \mathrm{IC}_{50}$ dose of $\mathbf{1 6}$. On the contrary, cells treated with 14 behaved similarly to control cells even at the highest concentration tested $(16.66 \mu \mathrm{M})$, reinforcing the idea that compound $\mathbf{1 4}$ is less bioactive than $\mathbf{1 6}$.

The difference between glycolytic capacity and glycolysis rate defines glycolytic reserve. This parameter was also significantly diminished at $2 \times \mathrm{IC}_{50}$ dose of $\mathbf{1 6}$.

From these assays, it can be concluded that in the presence of 16, glycolysis of SW-620 cancer cells as energetic pathway is diminished (reduced glycolysis rate, glycolytic capacity, and glycolytic reserve).

We next analyzed mitochondrial respiration by monitoring the OCR using a mitostress standard assay (Fig. 3). For this purpose, SW-620 cells were treated for 48 hours with compound 16 at $0.83 \mu \mathrm{M}=\mathrm{IC}_{50}$ and $1.66 \mu \mathrm{M}=2 \times \mathrm{IC}_{50}$, and with

TABLE 1

Inhibitory effects of test compounds on SW-620 human colon cancer cell viability

Data are the mean \pm S.E.M. of at least three independent experiments, each performed in triplicate. Data $(>100)$; not significant activity found at $150 \mu \mathrm{M}$ concentration, after 48-hour treatment.

\begin{tabular}{lcccc}
\hline \multicolumn{1}{c}{ Compound } & $\mathrm{IC}_{50}(\mu \mathrm{M})^{a}$ & $\mathrm{GI}_{50}(\mu \mathrm{M})^{b}$ & $\mathrm{TGI}(\mu \mathrm{M})^{c}$ & $\mathrm{LC}_{50}(\mu \mathrm{M})^{d}$ \\
\hline $\mathbf{1 3}$ & $>100$ & $>100$ & $>100$ & $>100$ \\
$\mathbf{1 4}$ & $29.5 \pm 10.18$ & $10.5 \pm 1.55$ & $56.25 \pm 7.46$ & $75.5 \pm 5.42$ \\
$\mathbf{1 5}$ & $2 \pm 0$ & $1.58 \pm 0.08$ & $10.33 \pm 1.45$ & $19.33 \pm 2.85$ \\
$\mathbf{1 6}$ & $0.83 \pm 0.083$ & $0.57 \pm 0.067$ & $14.47 \pm 12.77$ & $75 \pm 25$ \\
$\mathbf{1 7}$ & $2.67 \pm 0.67$ & $1.83 \pm 0.083$ & $>100$ & $>100$ \\
$\mathbf{2 0}$ & $43.33 \pm 4.41$ & $24.33 \pm 8.29$ & $>100$ & $>100$ \\
$\mathbf{2 4}$ & $5.17 \pm 1.17$ & $2.33 \pm 0.33$ & $36.67 \pm 4.41$ & $63.33 \pm 8.33$ \\
SF-6 & $>100$ & $>100$ & $>100$ & $>100$ \\
$\mathbf{4 , 4}$-Di-O-MEA & $5.97 \pm 1.09$ & $1.58 \pm 0.18$ & $143.3 \pm 23.66$ & $>100$ \\
\hline
\end{tabular}

4,4'-Di- $O$-MEA, 4,4'-di- $O$-methylellagic acid.

${ }^{a}$ Effective concentration, or compound concentration required for $50 \%$ inhibition of cell proliferation, after 48 -hour treatment.

${ }^{b}$ Compound concentration required for $50 \%$ cell growth inhibition, after 48 -hour treatment.

${ }^{c}$ Compound concentration required for total cell growth inhibition, after 48-hour treatment.

${ }^{d}$ Compound concentration required for $50 \%$ cell death, after 48 -hour treatment. 
TABLE 2

Sensitivity of SW-480 and SW-620 human colon cancer cells to compound $\mathbf{1 6}$

Data are the mean \pm S.E.M. of at least three independent experiments, each performed in triplicate.

\begin{tabular}{lcccc}
\hline Colorectal Cancer Cell Line & $\mathrm{IC}_{50}(\mu \mathrm{M})^{a}$ & $\mathrm{GI}_{50}(\mu \mathrm{M})^{b}$ & $\mathrm{TGI}(\mu \mathrm{M})^{c}$ & $\mathrm{LC}_{50}(\mu \mathrm{M})^{d}$ \\
\hline SW-480 & $4.03 \pm 0.183$ & $2.67 \pm 0.27$ & $24.31 \pm 15.32$ & $84 \pm 17$ \\
SW-620 & $0.83 \pm 0.083$ & $0.57 \pm 0.067$ & $14.47 \pm 12.77$ & $75 \pm 25$ \\
\hline
\end{tabular}

${ }^{a}$ Effective concentration, or compound concentration required for $50 \%$ inhibition of cell proliferation, after 48 -hour treatment.

${ }^{b}$ Compound concentration required for $50 \%$ cell growth inhibition, after 48-hour treatment.

${ }^{c}$ Compound concentration required for total cell growth inhibition, after 48-hour treatment.

${ }^{d}$ Compound concentration required for $50 \%$ cell death, after 48 -hour treatment.

compound 14 at 0.83 and $16.66 \mu \mathrm{M}$. Nontreated cells were kept as controls.

Basal respiration, that is, the energetic demand of the cells under baseline conditions, was measured first. As shown in Fig. 3A, cells treated with compound $\mathbf{1 6}$ at both concentrations displayed a reduced basal respiration compared with 14-treated cells and control cells.

All other parameters of mitochondrial function-ATP production, spare respiratory capacity, and proton leak-were determined following sequential injections of specific inhibitors: oligomycin (inhibitor of ATP synthase), carbonyl FCCP, a disrupter of mitochondrial membrane potential, and rotenone/antimycin A (inhibitors of complex I and III of electron transport chain) (Pike Winer and Wu, 2014).

OCR before oligomycin injection minus OCR after oligomycin injection determines the portion of oxygen dedicated to ATP production by mitochondria. As shown in Fig. 3A, this parameter is significantly reduced in 16-treated cells at $2 \times \mathrm{IC}_{50}$ concentration compared with 14-treated cells and control nontreated cells.

Next, FCCP, which mimics an increased physiologic energy demand, was injected to determine the maximal respiration rate or spare respiratory capacity. This parameter determines the amount of extra ATP that can be produced by the cell in case of a sudden increase of energy demand as well as how closely the cell is breathing to its theoretical maximum. As it can be observed in Fig. 3A, after adding FCCP, cells treated with compound $\mathbf{1 6}$ at $2 \times \mathrm{IC}_{50}$ dose displayed a significant reduction on the reserve respiratory capacity parameter compared with 14-treated cells and control nontreated cells.
Finally, antimycin A and rotenone were added to completely inhibit mitochondrial respiration. The difference between OCR after oligomycin injection and OCR after rotenone and antimycin A injection was used to determine the proton leak function. This parameter determines the remaining basal respiration not devoted to ATP production and can be a sign of mitochondrial damage. As it can be observed in Fig. 3A, cells displayed a significant reduction on proton leak at all tested concentrations of 16- compared with compound 14-treated cells and control nontreated cells.

Next, we run a similar assay to compare mitochondrial respiration of 16- and 14-treated cells at $2 \times \mathrm{IC}_{50}$ dose of each compound, this is $1.66 \mu \mathrm{M}$ for 16 and $60 \mu \mathrm{M}$ for 14. As it is shown in Fig. 3B, 14 at $60 \mu \mathrm{M}$ diminished mitochondrial function in a similar way than 16 at $1.66 \mu \mathrm{M}$. These results indicate that $\mathbf{1 4}$ is indeed effective in targeting mitochondrial respiration, but it requires a higher concentration than $16(60 \mu \mathrm{M}$ for 14 versus $1.66 \mu \mathrm{M}$ for 16) in accordance with the previously determined $\mathrm{IC}_{50}$ value.

The discovery that compound $\mathbf{1 6}$ affects cell bioenergetics in colon cancer cells allows us to propose that its anticancer activity might be due, at least in part, to this property. It should be highlighted that, different from other scenarios in which the energetic metabolism is rewired from glycolysis to mitochondrial respiration (Dai et al., 2015) or the other way around (Tesori et al., 2015), treatment with 16 diminishes both.

AMPK Activation. As it was previously mentioned, AMPK is an energy sensor that regulates energy metabolism. This is a heterotrimeric Ser/Thr kinase complex characterized

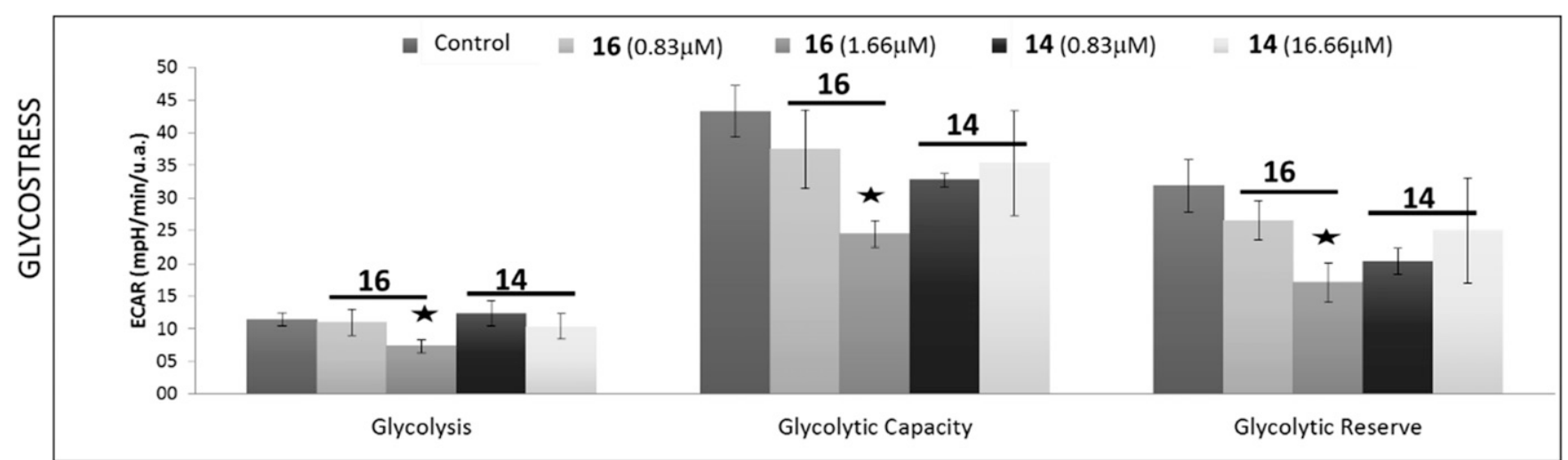

Fig. 2. Glycolytic activity in SW-620 colon cancer cells. Comparison of the glycolytic function in SW-620-nontreated cells versus 16-treated cells (0.83 and $1.66 \mu \mathrm{M})$ and 14-treated cells $(0.83$ and $16.66 \mu \mathrm{M})$. Glycolysis (difference from ECAR value in the presence of glucose and ECAR value in starved cells), glycolytic reserve (difference between ECAR after ATPase inhibition and ECAR after glucose injection), and glycolytic capacity (sum of glycolysis rate and glycolytic reserve) are shown. Representative assay of two experiments. Each experiment contains six replicates per treatment. Asterisk indicates statistical differences in treated cells with respect to the control (nontreated cells) $* P<0.05$. 


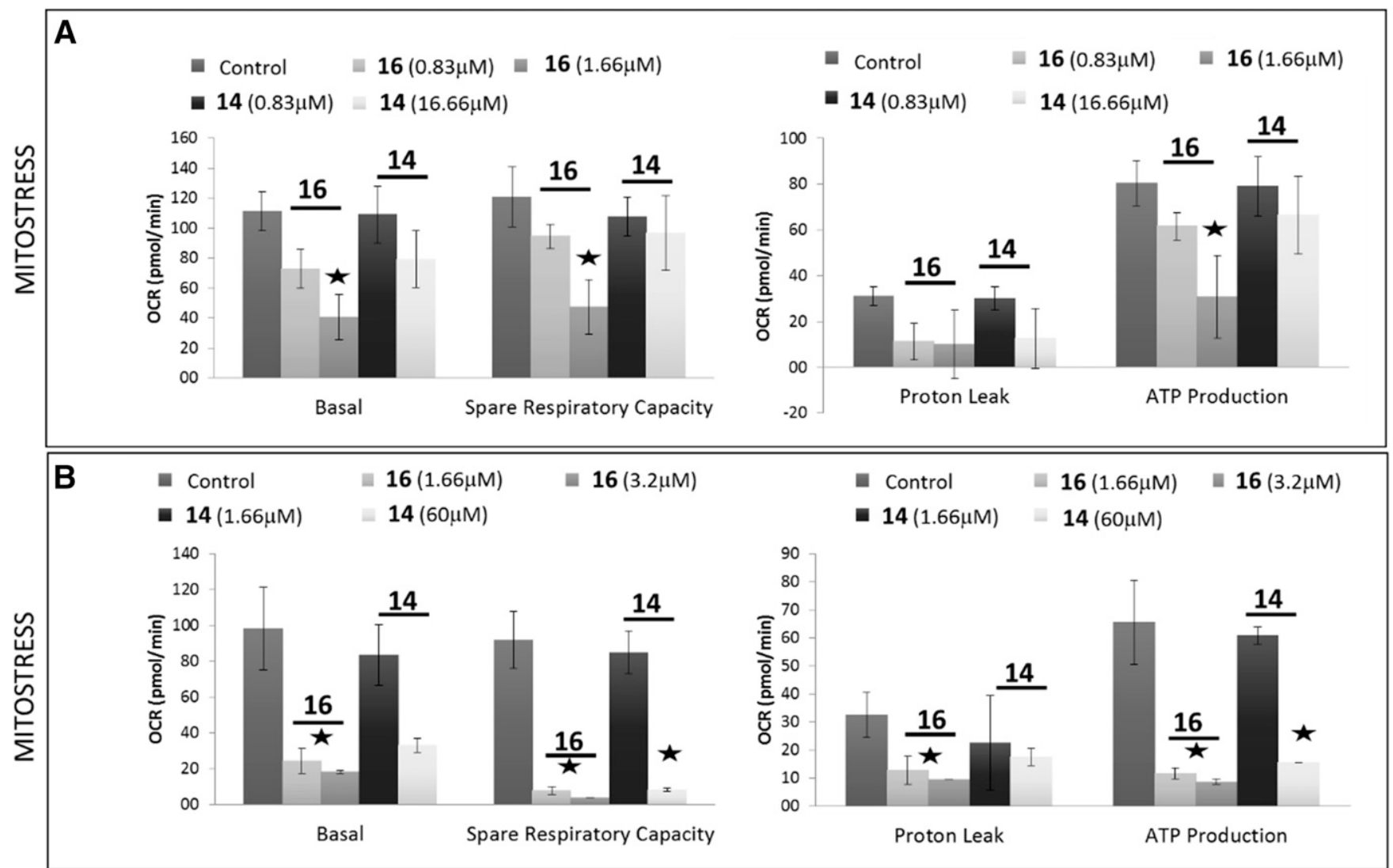

Fig. 3. Mitochondrial respiration in SW-620 colon cancer cells. (A) Mitochondrial respiration analysis by flux analysis of the OCR in SW-620-nontreated cells versus 16-treated cells $(0.83$ and $1.66 \mu \mathrm{M})$ and 14-treated cells $(0.83$ and $16.66 \mu \mathrm{M})$. (B) Mitochondrial respiration analysis by flux analysis of the OCR in SW-620-nontreated cells versus 16-treated cells (1.66 and 3.2 $\mu \mathrm{M})$ and 14-treated cells (1.66 and $60 \mu \mathrm{M})$. Representative assays of two experiments. Each experiment contains six replicates per treatment. Asterisk indicates statistical differences in treated cells with respect to the control (nontreated cells) $* P<0.05$.

by a catalytic $\alpha$ subunit and two regulatory subunits $(\beta, \gamma)$ (Zadra et al., 2015). For the full activity of the kinase, a phosphorylation at the residue $\mathrm{Thr}_{172}$ in the catalytic loop is required. The active phosphorylated form of AMPK (P-AMPK) inhibits essentially all anabolic pathways that promote cell growth (Faubert et al., 2013). Thus, in cancer in which the energy demands of the cell are elevated, AMPK activators may suppress tumor growth. The role of AMPK as a tumor suppressor is supported by the observation that patients on treatment with metformin, an activator of AMPK, have a lower cancer incidence (Evans et al., 2005; Vazquez-Martin et al., 2009).

Due to the critical role of AMPK in the regulation of energy and redox homeostasis, we wanted to check whether compound
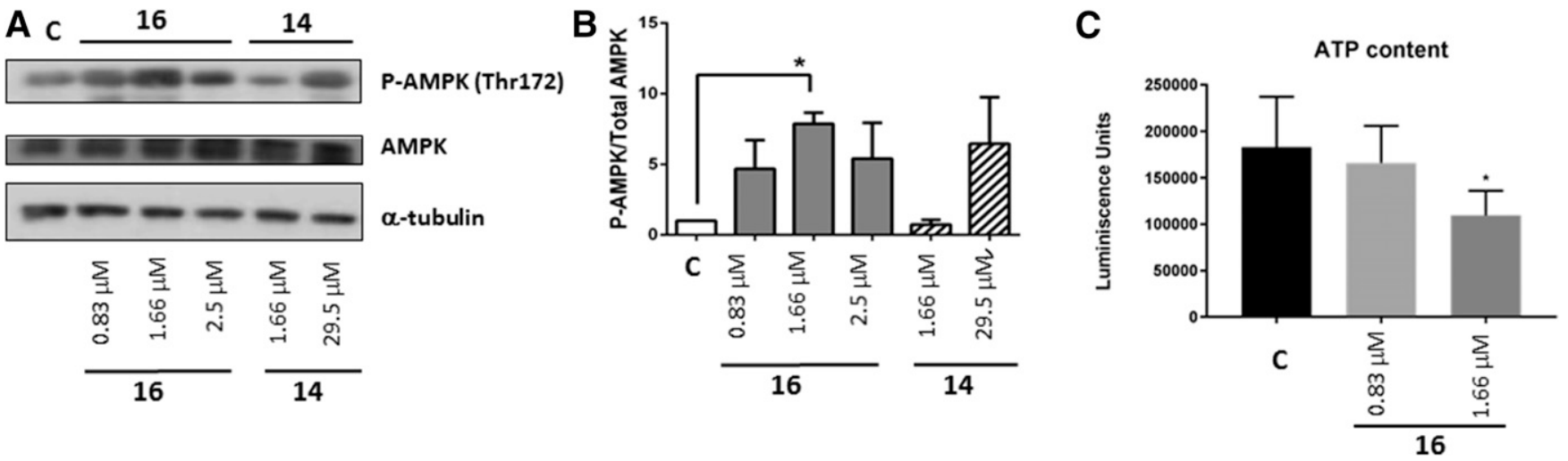

Fig. 4. Compound 16 leads to AMPK activation. (A) Western blot analysis of P-(Thr $\left.{ }_{172}\right)$ AMPK and total AMPK. SW-620 cells were treated for 48 hours with compound $16(\mathrm{~d} 1: 0.83 \mu \mathrm{M} ; \mathrm{d} 2: 1.66 \mu \mathrm{M}$; and d3: $2.5 \mu \mathrm{M})$ and $\mathbf{1 4}(\mathrm{d} 1: 1.66 \mu \mathrm{M}$ and d2: $29.5 \mu \mathrm{M})$. Representative experiment. (B) Western blot quantification of ratio P-(Thr $\left.{ }_{172}\right)$ AMPK/total AMPK. (C) Measurement of the intracellular ATP content. Luminiscence units were registered for compound 16-treated cells $(0.83$ and $1.66 \mu \mathrm{M})$ and compared with control nontreated cells. Asterisk indicates statistical differences in treated cells with respect to the control (nontreated cells) $* P<0.05$. 
16 can contribute to its activation. As shown in Fig. 4A, treatment of colon cancer cells with compound 16, even at the lowest concentration $(0.83 \mu \mathrm{M})$, significantly increased the amount of active $\mathrm{P}-\mathrm{AMPK}\left(\mathrm{Thr}_{172}\right)$. Consequently, the ratio P-AMPK(Thr $\left.{ }_{172}\right) /$ total AMPK(Fig. 4B) is higher for 16 compared with control cells. Importantly, compound 14 was also able to activate AMPK, but it required a higher concentration than $16(29.5 \mu \mathrm{M}$ for 14 versus $1.66 \mu \mathrm{M}$ for 16) (Fig. 4, A and B). These results indicate that the two structurally related polyphenols, 16 and 14, are able to activate AMPK, but 16 was 18 times more potent than 14.

As SW-620 cells treated with 16 showed defective mitochondrial ATP production, together with decreased aerobic glycolysis, leading to AMPK activation, we analyzed the effect of this compound on the cellular ATP content. For this purpose, colorectal cancer SW-620 cells were treated for 48 hours with compound $\mathbf{1 6}$ at two concentrations $(0.83 \mu \mathrm{M}$ $=\mathrm{IC}_{50}$ and $1.66 \mu \mathrm{M}=2 \times \mathrm{IC}_{50}$ ). Then 10,000 viable cells of nontreated and treated cells were replated. After 6 hours in complete media, without treatments, the ATP content was measured by means of the ATP-based assay CellTiter-Glo Luminescent Cell Viability kit (Promega). As shown in Fig. $4 \mathrm{C}$, the cellular ATP content was significantly reduced with respect to the control (nontreated cells) in the presence of the highest concentration of $16(1.66 \mu \mathrm{M})(P<0.038)$. From this study, we concluded that compound $\mathbf{1 6}$ diminishes cell bioenergetics, leading to AMPK activation as a sensor of ATP depletion.

Induction of Apoptosis. Finally, as compound 16 inhibited cell viability, diminishing cell bioenergetics, and activating AMPK, we wanted to determine whether it might induce apoptosis.

Apoptosis is a vital biologic process of multicellular organisms by which damaged, mutant, and aged cells are eliminated in a programmed way. As previously mentioned, a variety of natural polyphenols, like curcumin or resveratrol, induces apoptotic cell death in a variety of cancer models. This process is mediated by the activation of some specific proteases named caspases (Reed, 2000; Oliver and Vallette, 2005). Apoptosis, mediated by caspase activation, may result in induction of death in cancerous cells and therefore represent a promising strategy to develop cancer chemotherapeutics.

There are more than 13 known caspases (procaspases or active cysteine caspases) that can be detected using various types of caspase activity assays (Nicholson and Thornberry, 1997; Thornberry et al., 1997; Thornberry and Lazebnik, 1998; García-Calvo et al., 1999; Bayascas et al., 2002; Le et al., 2002; Mooney et al., 2002). Among them, caspase-3 and -7 are especially important because they play a key role in the apoptotic pathway. In fact, their activation is being considered as a typical hallmark of apoptosis.

In the present study, we wanted to check whether induction of apoptosis could also be implicated in the observed cell growth inhibition promoted by 16. With this purpose, the activity of caspase 3 and 7 was monitored using a luminescent assay. For this assay, a time period of 48 hours has been chosen because in a previous experiment carried out to determine the effect of the tested compounds on cell viability at different times $(24,48$, and 72 hours), it was found that 24 hours is a period of time too short to induce cellular lethality, whereas 72 hours is too long, ending with the cell death of most cells (data not shown).

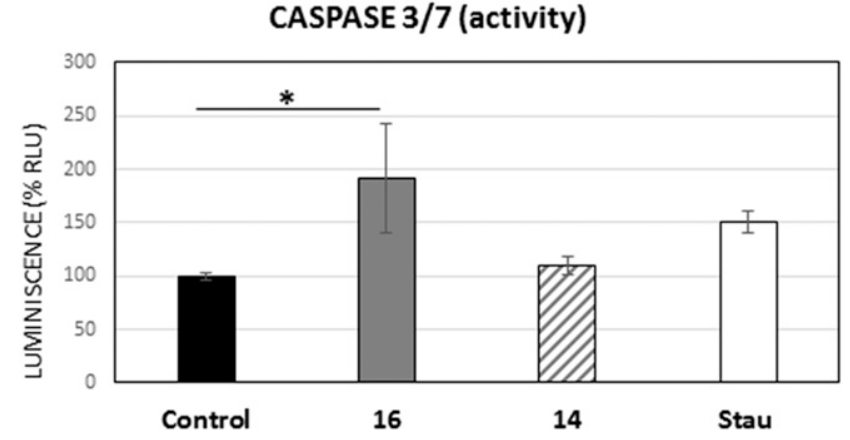

Fig. 5. Caspase $3 / 7$ activity. Activation of caspase 3 and 7 after 48 -hour treatment with compounds $14(1.66 \mu \mathrm{M})$ and $16(1.66 \mu \mathrm{M})$. SW-620 cells treated with staurosporine $(1.5 \mu \mathrm{M})$ were used as the positive control. Data represent means \pm S.E.M. of two independent experiments, each performed in triplicate. Asterisks indicate statistical differences in treated cells with respect to the control (nontreated cells) $* P<0.05$.

Thus, SW-620 cells were treated for 48 hours with compounds 14 and 16, both at $1.66 \mu \mathrm{M}$ (twofold of the $\mathrm{IC}_{50}$ shown for compound 16). Staurosporine $(1.5 \mu \mathrm{M})$-treated cells were used as positive control of caspase $3 / 7$ activation. Staurosporine is an alkaloid isolated from Streptomyces staurosporeus known to activate apoptosis in several cancer cells (Yadav et al., 2015).

As it is shown in Fig. 5, activity of caspase 3/7 after 48 hours was significantly increased in SW-620-treated cells with compound 16 compared with control and 14. Remarkably, higher effect on caspase 3/7 activation was also observed with $\mathbf{1 6}$ compared with the positive control, staurosporine.

Our results showed that compound $\mathbf{1 6}$ is able to stimulate significantly caspase activation in SW-620 cancer cells.

\section{Discussion}

Although metabolic alteration is one of the oldest mechanisms associated with cancer development, only recently its targeting became a promising anticancer strategy. In fact, altered metabolism is now considered an emerging hallmark of cancer, and researches in the field hope to find drugs that may lead to a new class of anticancer drugs (Jang et al., 2013). However, this field is in a very preliminary stage, and the effective number of compounds under investigation as potential modulators of cell metabolism is still very poor. For this reason, the identification of metabolically active agents can be of great interest for anticancer therapies.

In this study, we provide evidence of the inhibitory effects on colon cancer growth and metabolism of a new family of synthetic polyphenols. These compounds have a hydrophilic head, composed of two galloyl (3,4,5-trihydroxybenzoyl) units, and a hydrophobic tail linked through a serinol moiety. They were very efficiently obtained (high yields and easy purifications) in three steps by $N$-acylation of serinol, followed by coupling with MOM-protected gallic acid and deprotection with aqueous hydrochloric acid.

On the basis of the structure-activity-relationship studies performed on this family, it appears that the presence of the two galloyl moieties at the same side of the molecules is essential for inhibitory growth, and that the length of the $N$ acyl chain is also crucial for activity, compounds with $\mathrm{C} 12$ and C18 chain length being the best. A decrease of the acyl chain 
length, reduction of the number of $\mathrm{OHs}$ on the aromatic ring, or substitution of serinol by ethanolamine as linker lowers or eliminates their anticancer properties. However, the introduction of a double bond on the hydrophobic tail increases the inhibitory growth properties. Compound 16, with an unsaturated $\mathrm{C} 18$ chain, showed the highest antiproliferative effect in SW-620 colon cancer cells.

Our results indicate that compound $\mathbf{1 6}$ reduces the energetic metabolism (glycolysis and mitochondrial respiration) in SW-620 colon cancer cells at the same time that it activates AMPK and caspase 3 and 7 activity. All of these findings lead us to suggest that, through AMPK activation, compound $\mathbf{1 6}$ might lead to alterations in colon cancer cell bioenergy compromising cancer cell viability. Importantly, these antiproliferative and proapoptotic effects have been shown to be selective for cancer cells, indicating a therapeutic window for the use of this compound.

Of particular interest is the simultaneous inhibition of the two main energy-producing pathways (glycolysis and oxidative phosphorylation) shown by $\mathbf{1 6}$ in colon cancer cells. In this respect, it has been postulated recently that the pharmacological inhibition of several complementary metabolic pathways (antimetabolic cooperativity) can be better to eliminate cancer cells than the inhibition of a single one (Marchetti et al., 2015).

\section{Conclusion}

As recently emphasized by Jang et al. (2013), cancer cell metabolism has become one of the most exciting and promising fields for the development of new anticancer agents. Targeting cancer metabolism opens an opportunity to develop broadly applicable drugs that can treat multiple cancer types, and hence may lead to a new class of anticancer drugs.

We concluded that the novel molecules described in this work, and in particular 16, the most potent within this series, show antiproliferative properties in two different colon carcinoma cell lines (SW-480 and SW-620) and reduce the energetic metabolism (glycolysis and mitochondrial respiration) in SW-620 colon cancer cells at the same time that they activate AMPK and caspase 3 and 7 activity. Based on that, 16 and its analogs could serve as hits to develop a new class of therapeutic agents that target tumor metabolism. Of particular interest is the simultaneous inhibition of the two main energy-producing pathways (glycolysis and oxidative phosphorylation) shown by $\mathbf{1 6}$ in colon cancer cells. Further research is needed to fully elucidate the possible mechanism(s) of action to suppress tumor growth. This is a nontrivial task because many different metabolic enzymes, oncogenic signaling pathways, and/or regulatory network can be implied.

\section{Acknowledgments}

We acknowledge M. A. Bonache for help with the processing of supporting information.

\section{Authorship Contributions}

Participated in research design: San-Félix, Pérez-Pérez, Quintela, Gómez de Cedrón, Ramírez de Molina, Reglero.

Conducted experiments: Madrona, Jiménez, Vargas, Gómez de Cedrón.

Performed data analysis: San-Félix, Gómez de Cedrón.

Wrote or contributed to the writing of the manuscript: San-Félix, Gómez de Cedrón.

\section{References}

Arts IC and Hollman PC (2005) Polyphenols and disease risk in epidemiologic studies. Am J Clin Nutr 81 (Suppl):317S-325S.

Bayascas JR, Yuste VJ, Benito E, Garcia-Fernàndez J, and Comella JX (2002) Isolation of AmphiCASP-3/7, an ancestral caspase from amphioxus (Branchiostoma floridae): evolutionary considerations for vertebrate caspases. Cell Death Differ $\mathbf{9}$ 1078-1089.

Belin FBP, Ruiz K, Lacombe JM, and Pucci B (2003) Synthetic gallic acid derivatives as models for a comprehensive study of antioxidant activity. Helv Chim Acta $\mathbf{8 6}$ : 247-265

Bieberich E, Hu B, Silva J, MacKinnon S, Yu RK, Fillmore H, Broaddus WC, and Ottenbrite RM (2002a) Synthesis and characterization of novel ceramide analogs for induction of apoptosis in human cancer cells. Cancer Lett 181:55-64.

Bieberich E, Hu B, Silva J, Mackinnon S, Yu RK, and Ottenbrite RM (2002b) Novel ceramide analogs are new anti-cancer drugs. Polym Preprint 43:756-757.

Bieberich E, Ottenbrite RM, Yu RK, Fillmore H, and Broaddus WC (2002c) inventors, Virginia Commonwealth University, assignee. Hydroxyalkyl amide analogs of ceramide. U.S. patent 6410597B1 Jun 25, 2002.

Boyer JZ, Jandova J, Janda J, Vleugels FR, Elliott DA, and Sligh JE (2012) Resveratrol-sensitized UVA induced apoptosis in human keratinocytes through mitochondrial oxidative stress and pore opening. $J$ Photochem Photobiol B 113: $42-50$.

Cerella C, Radogna F, Dicato M, and Diederich M (2013) Natural compounds as regulators of the cancer cell metabolism. Int J Cell Biol 2013:639401.

Dai W, Wang F, Lu J, Xia Y, He L, Chen K, Li J, Li S, Liu T, Zheng Y, et al. (2015) By reducing hexokinase 2, resveratrol induces apoptosis in HCC cells addicted to aerobic glycolysis and inhibits tumor growth in mice. Oncotarget 6:13703-13717.

Evans JM, Donnelly LA, Emslie-Smith AM, Alessi DR, and Morris AD (2005) Metformin and reduced risk of cancer in diabetic patients. BMJ 330:1304-1305.

Faubert B, Boily G, Izreig S, Griss T, Samborska B, Dong Z, Dupuy F, Chambers C, Fuerth BJ, Viollet B, et al. (2013) AMPK is a negative regulator of the Warburg effect and suppresses tumor growth in vivo. Cell Metab 17:113-124.

Flores A, Camarasa MJ, Pérez-Pérez MJ, San-Félix A, Balzarini J, and Quesada E (2014) Multivalent agents containing 1-substituted 2,3,4-trihydroxyphenyl moieties as novel synthetic polyphenols directed against HIV-1. Org Biomol Chem 12: 5278-5294.

Garcia-Calvo M, Peterson EP, Rasper DM, Vaillancourt JP, Zamboni R, Nicholson DW, and Thornberry NA (1999) Purification and catalytic properties of human caspase family members. Cell Death Differ 6:362-369.

Gažák R, Valentová K, Fuksová K, Marhol P, Kuzma M, Medina MA, Oborná I, Ulrichová J, and Krren V (2011) Synthesis and antiangiogenic activity of new silybin galloyl esters. J Med Chem 54:7397-7407.

González-Vallinas M, González-Castejón M, Rodríguez-Casado A, and Ramírez de Molina A (2013) Dietary phytochemicals in cancer prevention and therapy: a complementary approach with promising perspectives. Nutr Rev 71:585-599.

Hadi SM, Bhat SH, Azmi AS, Hanif S, Shamim U, and Ullah MF (2007) Oxidative breakage of cellular DNA by plant polyphenols: a putative mechanism for anticancer properties. Semin Cancer Biol 17:370-376.

Hardie DG (2014) AMP-activated protein kinase: maintaining energy homeostasis at the cellular and whole-body levels. Annu Rev Nutr 34:31-55.

Hewitt RE, McMarlin A, Kleiner D, Wersto R, Martin P, Tsokos M, Stamp GWH, and Stetler-Stevenson WG (2000) Validation of a model of colon cancer progression [published correction appears in J Pathol (2001) 194:507]. J Pathol 192: $446-454$

Hung CM, Su YH, Lin HY, Lin JN, Liu LC, Ho CT, and Way TD (2012) Demethoxycurcumin modulates prostate cancer cell proliferation via AMPK-induced downregulation of HSP70 and EGFR. J Agric Food Chem 60:8427-8434.

Jang M, Kim SS, and Lee J (2013) Cancer cell metabolism: implications for therapeutic targets. Exp Mol Med 45:e45.

Kim MY, Trudel LJ, and Wogan GN (2009) Apoptosis induced by capsaicin and resveratrol in colon carcinoma cells requires nitric oxide production and caspase activation. Anticancer Res 29:3733-3740.

Kubatka P, Kapinová A, Kello M, Kruzliak P, Kajo K, Výbohová D, Mahmood S, Murin R, Viera T, Mojžiš J, et al. (2016) Fruit peel polyphenols demonstrate substantial anti-tumour effects in the model of breast cancer. Eur J Nutr 55:955-965.

Landau EM and Siegel JS (2014) inventors, Universität Zürich, assignee. Lipidic biomaterials for encapsulation and triggered release., WO2014056939 Apr 17, 2014

Le DA, Wu Y, Huang Z, Matsushita K, Plesnila N, Augustinack JC, Hyman BT, Yuan J, Kuida K, Flavell RA, et al. (2002) Caspase activation and neuroprotection in caspase-3-deficient mice after in vivo cerebral ischemia and in vitro oxygen glucose deprivation. Proc Natl Acad Sci USA 99:15188-15193.

Li W, Saud SM, Young MR, Chen G, and Hua B (2015) Targeting AMPK for cancer prevention and treatment. Oncotarget 6:7365-7378.

López-Lázaro M (2010) A new view of carcinogenesis and an alternative approach to cancer therapy. Mol Med 16:144-153.

Luo Z, Zang M, and Guo W (2010) AMPK as a metabolic tumor suppressor: control of metabolism and cell growth. Future Oncol 6:457-470.

Marchetti P, Guerreschi P, Kluza J, and Mortier L (2014) Metabolic features of melanoma: a gold mine of new therapeutic targets? Curr Cancer Drug Targets 14: 357-370.

Marchetti P, Guerreschi P, Mortier L, and Kluza J (2015) Integration of mitochondrial targeting for molecular cancer therapeutics. Int J Cell Biol 2015:283145.

Merg AD, Slocik J, Blaber MG, Schatz GC, Naik R, and Rosi NL (2015) Adjusting the metrics of 1-D helical gold nanoparticle superstructures using multivalent peptide conjugates. Langmuir 31:9492-9501.

Mooney LM, Al-Sakkaf KA, Brown BL, and Dobson PR (2002) Apoptotic mechanisms in T47D and MCF-7 human breast cancer cells. Br J Cancer 87:909-917.

Neuhouser ML (2004) Dietary flavonoids and cancer risk: evidence from human population studies. Nutr Cancer 50:1-7. 
Nicholson DW and Thornberry NA (1997) Caspases: killer proteases. Trends Biochem Sci 22:299-306.

Oette K and Tschung TS (1980) [On the biosynthesis of glyceride- and phosphoglyceride analogues from monoglyceride analogue 2-(linoleoylamino)-1,3propanediol in rats (author's transl)]. Hoppe Seylers $Z$ Physiol Chem $\mathbf{3 6 1}$ 1179-1191.

Oliver L and Vallette FM (2005) The role of caspases in cell death and differentiation. Drug Resist Updat 8:163-170.

Osornio YM, Uebelhart P, Bosshard S, Konrad F, Siegel JS, and Landau EM (2012) Design and synthesis of lipids for the fabrication of functional lipidic cubic-phase biomaterials. J Org Chem 77:10583-10595.

Pelicano H, Martin DS, Xu RH, and Huang P (2006) Glycolysis inhibition for anticancer treatment. Oncogene 25:4633-4646.

Pierini R, Gee JM, Belshaw NJ, and Johnson IT (2008) Flavonoids and intestinal cancers. Br J Nutr 99 (E Suppl 1): ES53-ES59.

Pike Winer LS and Wu M (2014) Rapid analysis of glycolytic and oxidative substrate flux of cancer cells in a microplate. PLoS One 9:e109916.

Ramírez de Molina A, Vargas T, Molina S, Sánchez J, Martínez-Romero J, GonzálezVallinas M, Martín-Hernández R, Sánchez-Martínez R, Gómez de Cedrón M, Dávalos A, et al. (2015) The ellagic acid derivative 4,4'-di-O-methylellagic acid efficiently inhibits colon cancer cell growth through a mechanism involving WNT16. J Pharmacol Exp Ther 353:433-444.

Rashmi R, Santhosh Kumar TR, and Karunagaran D (2003) Human colon cancer cells differ in their sensitivity to curcumin-induced apoptosis and heat shock protects them by inhibiting the release of apoptosis-inducing factor and caspases. FEBS Lett 538:19-24.

Reed JC (2000) Mechanisms of apoptosis. Am J Pathol 157:1415-1430.

Rivero-Buceta E, Carrero P, Casanova E, Doyagüez EG, Madrona A, Quesada E Peréz-Pérez MJ, Mateos R, Bravo L, Mathys L, et al. (2015a) Anti-HIV-1 activity of a tripodal receptor that recognizes mannose oligomers. Eur J Med Chem 106 132-143.

Rivero-Buceta E, Carrero P, Doyagüez EG, Madrona A, Quesada E, Camarasa MJ Peréz-Pérez MJ, Leyssen P, Paeshuyse J, Balzarini J, et al. (2015b) Linear and branched alkyl-esters and amides of gallic acid and other (mono-, di- and tri-) hydroxy benzoyl derivatives as promising anti-HCV inhibitors. Eur J Med Chem 92:656-671.

Sagnella SM, Conn CE, Krodkiewska I, and Drummond CJ (2011) Nonionic diethanolamide amphiphiles with unsaturated C18 hydrocarbon chains: thermotropic and lyotropic liquid crystalline phase behavior. Phys Chem Chem Phys 13: 13370-13381.

Schulz TC and Robins AJ (2004) inventors, University of Georgia Research Foundation (UGARF), assignee. Neural differentiation of human embryonic stem cells using protease passaging techniques, and applications in treating neural disorders. WO2004090096,A2 U.S, patent 10121 Oct 21, 2004

Shin SM, Cho IJ, and Kim SG (2009) Resveratrol protects mitochondria against oxidative stress through AMP-activated protein kinase-mediated glycogen synthase kinase-3beta inhibition downstream of poly(ADP-ribose)polymerase-LKB1 pathway. Mol Pharmacol 76:884-895.

Teiten MH, Eifes S, Dicato M, and Diederich M (2010) Curcumin: the paradigm of a multi-target natural compound with applications in cancer prevention and treatment. Toxins (Basel) 2:128-162.

TeSlaa T and Teitell MA (2014) Techniques to monitor glycolysis. Methods Enzymol 542:91-114.

Tesori V, Piscaglia AC, Samengo D, Barba M, Bernardini C, Scatena R, Pontoglio A Castellini L, Spelbrink JN, Maulucci G, et al. (2015) The multikinase inhibitor Sorafenib enhances glycolysis and synergizes with glycolysis blockade for cancer cell killing. Sci Rep 5:9149.

Thornberry NA and Lazebnik Y (1998) Caspases: enemies within. Science 281: 1312-1316.

Thornberry NA, Rano TA, Peterson EP, Rasper DM, Timkey T, Garcia-Calvo M, Houtzager VM, Nordstrom PA, Roy S, Vaillancourt JP, et al. (1997) A combinatorial approach defines specificities of members of the caspase family and granzyme B: functional relationships established for key mediators of apoptosis. J Biol Chem 272:17907-17911.

Vázquez-Martín A, Oliveras-Ferraros C, López-Bonet E, and Menéndez JA (2009) AMPK: evidence for an energy-sensing cytokinetic tumor suppressor. Cell Cycle 8: $3679-3683$

Warburg O (1956) On the origin of cancer cells. Science 123:309-314.

Yadav SS, Prasad CB, Prasad SB, Pandey LK, Singh S, Pradhan S, and Narayan G (2015) Anti-tumor activity of staurosporine in the tumor microenvironment of cervical cancer: an in vitro study. Life Sci 133:21-28.

Zadra G, Batista JL, and Loda M (2015) Dissecting the dual role of AMPK in cancer: from experimental to human studies. Mol Cancer Res 13:1059-1072.

Zhang Q, Cheng G, Qiu H, Zhu L, Ren Z, Zhao W, Zhang T, and Liu L (2015) The p53inducible gene 3 involved in flavonoid-induced cytotoxicity through the reactive oxygen species-mediated mitochondrial apoptotic pathway in human hepatoma cells. Food Funct 6:1518-1525.

Address correspondence to: Ana Ramírez de Molina, IMDEA Food Institute, Crta. Cantoblanco 8, 28049, Madrid, Spain. E-mail: ana.ramirez@ imdea.org; or Ana San-Félix, Instituto de Química Médica (IQM-CSIC), Juan de la Cierva 3, 28006, Madrid, Spain. E-mail: anarosa@iqm.csic.es 